\title{
Testing the forward modeling approach in asteroseismology
}

\section{Structure and internal dynamics of the hot B subdwarf component in the close eclipsing binary system PG 1336-018}

\author{
S. Charpinet ${ }^{1}$, V. Van Grootel ${ }^{1,2}$, D. Reese ${ }^{3}$, G. Fontaine ${ }^{2}$, E. M. Green ${ }^{4}$, P. Brassard ${ }^{2}$, and P. Chayer ${ }^{5}$ \\ ${ }^{1}$ Laboratoire d'Astrophysique de Toulouse-Tarbes, Université de Toulouse, CNRS, 14 Av. E. Belin, 31400 Toulouse, France \\ e-mail: [stephane.charpinet; valerie.vangrootel] @ast.obs-mip.fr \\ 2 Département de Physique, Université de Montréal, C.P. 6128, Succ. Centre-Ville, Montréal, QC H3C 3J7, Canada \\ e-mail: [fontaine; brassard]@astro.umontreal.ca \\ 3 Department of Applied Mathematics, University of Sheffield, Hounsfield Road, Sheffield, S3 7RH, UK \\ e-mail: D.Reese@sheffield.ac.uk \\ 4 Steward Observatory, University of Arizona, 933 North Cherry avenue, Tucson, AZ 85721, USA \\ e-mail: bgreen@as.arizona.edu \\ 5 Space Telescope Science Institute, 3700 San Martin Drive, Baltimore, MD 21218, USA \\ e-mail: chayer@stsci.edu
}

Received 4 April 2008 / Accepted 17 July 2008

\begin{abstract}
Aims. We present a stringent test on the forward modeling technique in asteroseismology by confronting the predictions of a detailed seismic analysis of the pulsating subdwarf component in the unique close eclipsing binary system PG 1336-018 with those derived independently from modeling the binary light curve of the system. We also take advantage of the observed rotationally-split rich period spectrum to investigate the internal dynamics of the pulsating component in this system expected to be tidally locked. Methods. We carry out numerical exercises based on the double optimization technique that we developed within the framework of the forward modeling approach in asteroseismology. We use a recently updated version that now incorporates the effects of stellar rotation on the pulsation properties. We thus search in parameter space for the optimal model that objectively leads to the best simultaneous match of the 25 periods (including rotationally-split components) observed in PG 1336-018. For the first time, we also attempt to precisely reconstruct the internal rotation profile of the pulsator from its oscillations.

Results. Our principal result is that our seismic model, which closely reproduces the observed periods, is remarkably consistent with one of the best-fitting possible solutions uncovered independently from the binary light curve analysis, in effect pointing to the correct one. The latter indicates a mass of $M_{*}=0.466 \pm 0.006 M_{\odot}$ and a radius of $R_{*}=0.15 \pm 0.01 R_{\odot}$ for the sdB star. In comparison, our seismic analysis, combined to high-quality time-averaged spectroscopy, leads to the following estimates of the basic structural parameters of the sdB component: $M_{*}=0.459 \pm 0.005 M_{\odot}, R_{*}=0.151 \pm 0.001 R_{\odot}, \log g=5.739 \pm 0.002, T_{\text {eff }}=32740 \pm 400 \mathrm{~K}$, and $\log \left(M_{\text {env }} / M_{*}\right)=-4.54 \pm 0.07$. We also find strong evidence that the sdB star has reached spin-orbit synchronism and rotates as a solid body down to at least $r \sim 0.55 R_{*}$. We further estimate that higher-order perturbation effects due to rotation and tidal deformation of the star are insufficient to alter in a significant way the proposed asteroseismic solution itself (i.e., the derived structural parameters and rotation properties). Future efforts to improve further the accuracy of the seismic models will clearly have to incorporate such effects, however.

Conclusions. We conclude that our approach to the asteroseismology of sdB stars has passed a fundamental test with this analysis of PG 1336-018. The structural parameters and inferences about the internal dynamics of this star derived in the present paper through this approach should rest on very solid grounds. More generally, our results underline the power and usefulness of the forward modeling method in asteroseismology, despite historical misgivings about it.
\end{abstract}

Key words. stars: binaries: close - stars: subdwarfs - stars: oscillations - stars: interiors - stars: rotation stars: individual: PG 1336-018

\section{Introduction}

The object PG 1336-018 $(V=13.45 \pm 0.09)$ was discovered in the course of the Palomar-Green survey where it was first classified as a hot B subdwarf (sdB) star (Green et al. 1986). Kilkenny et al. (1998) found out subsequently that PG 1336-018 is, in fact, a double system, one of the very few known $H W$ Vir-type $\mathrm{sdB}+$ dwarf $\mathrm{M}$ close eclipsing binaries, featuring deep eclipses and strong reflection effects. Its official IAU name then became $N Y$ Virginis. Only three other eclipsing binaries of this kind are currently known: HW Vir (PG 1241-084) itself (Menzies \& Marang 1986; Wood et al. 1993), HS 0705+6700 (Drechsel et al. 2001), and HS 2231+2441 (Østensen et al. 2007). One unique feature of PG 1336-018 among this very select class of stars, is that its $\mathrm{sdB}$ component, contrary to that of the other members of the class, is also a short-period pulsating star. The presence of pulsations in PG 1336-018 elevates this system to the status of a virtual Rosetta Stone of stellar astrophysics, offering the possibility for carrying out a basic test in stellar structure. Indeed, considering that asteroseismic and orbital 
modeling techniques can be used independently for accurate and concurrent assessments of important properties of the stellar components, it follows that a comparison of the results of these two approaches constitutes a fundamental consistency check between the two techniques.

Subdwarf B stars are found in the old disk (field sdBs), halo populations (globular cluster members), and in the bulge of our own Galaxy (Busso et al. 2005). In field surveys, they dominate the population of faint blue stars down to $V \sim 16$ and are now recognized as the most likely source for the UV-upturn phenomenon observed in old elliptical galaxies (Brown et al. 1997). They are hot and compact stars with atmospheric parameters in the ranges $20000 \mathrm{~K} \lesssim T_{\text {eff }} \lesssim 40000 \mathrm{~K}$ and $5.0 \lesssim \log g \lesssim 6.2$. The evolutionary status of sdB stars is now well established following their identification with models of Extreme Horizontal Branch (EHB) stars (Heber 1986; Saffer et al. 1994) that burn helium in their core and that have very thin H-rich residual envelopes (Dorman et al. 1993). However, major uncertainties still remain concerning the mechanisms that lead to the formation of such stars. While B subdwarfs undoubtedly evolved from the Red Giant Branch (RGB), they managed, unlike other classical horizontal branch stars, to loose all but a tiny fraction of their $\mathrm{H}$-rich envelope at the tip of the RGB, before or during the helium flash. Single star evolution would require enhanced and tuned mass loss on the RGB (D'Cruz et al. 1996) to produce EHB stars, a possibility that is deemed rather implausible nowadays as a major contributing channel to the whole population of sdB stars. Instead, focus has moved on binary evolution scenarios after recent observational surveys showed that a majority of sdB stars are found in binaries, with a significant fraction of them having nearby white dwarfs or M dwarf companions and showing orbital periods from hours to days (Green et al. 1997; Maxted et al. 2001; Morales-Rueda et al. 2003). The formation channels invoked include: 1) evolution via a common envelope (CE) that is ultimately ejected, leading to the formation of binaries with short orbital periods (between 0.1 and 10 days) and $\mathrm{sdB}$ stars with preferentially thin residual H-rich envelopes, 2) evolution through stable Roche lobe overflow (RLOF) resulting in binaries with somewhat longer orbital periods (10 to 100 days) and $\mathrm{sdB}$ stars having thicker $\mathrm{H}$-rich envelopes, and 3) mergers of two helium white dwarfs that result in single sdB stars. All these channels are expected to leave specific imprints not only on the binary distribution of hot B subdwarfs, but also on the structural properties of the produced sdB stars. In particular, each channel predicts a mass distribution for sdB stars that is strongly peaked at $\sim 0.46 M_{\odot}$ (i.e., close to the canonical mass value that is often assumed for these stars) but featuring more or less extended wings. The broader distribution results from the merger channel that could possibly produce sdB stars with masses as low as $0.3 M_{\odot}$ and as high as $0.7 M_{\odot}$ (see Han et al. 2002, 2003, and references therein).

Subdwarf B stars host two groups of nonradial pulsators. The first group - known as the V361 Hya or EC 14026 stars was discovered by Kilkenny et al. (1997) and independently predicted by Charpinet et al. (1996) on the basis of theoretical considerations. More than three dozen of these EC 14026 variables are now identified, all characterized by rapid oscillations with periods in the $80-600 \mathrm{~s}$ range. These pulsations are observed exclusively - but not systematically (only a minority of them show this behavior) - in the hottest sdB stars having $T_{\text {eff }} \sim 30000-36000 \mathrm{~K}$. The oscillations are caused by low-order, low-degree acoustic waves (or $p$-modes) driven by a $\kappa$-mechanism powered by local accumulations of iron due to radiative levitation, the latter being particularly efficient in the envelope of sdB stars (Charpinet et al. 1997, 2001). The second group of pulsators - named the V1093 Her, "Betsy stars", or PG $1716+426$ stars - was discovered more recently by Green et al. (2003) and is characterized by much longer oscillation periods in the 2000-9000 s range. These pulsations are observed exclusively - and, this time, almost systematically - in the coolest sdB stars with $T_{\text {eff }} \lesssim 30000 \mathrm{~K}$. In that case, the oscillations correspond to relatively high-order, low-degree gravity waves (or $g$-modes) driven by the same mechanism responsible for the pulsations in EC 14026 stars (Fontaine et al. 2003).

The EC 14026 pulsators have attracted particular attention these past few years because of their high potential in terms of probing sdB internal structures using asteroseismic techniques. The long period $g$-mode pulsators also hold very good promises for asteroseismology which, however, may be more difficult to exploit with our current modeling tools and ground-based observing facilities (but see the recent attempts conducted by Randall et al. 2005b, 2006b,c). Improvements in the models are currently being developed and observations from space, especially with the French satellite CoRoT, should also allow us to progress on this front in a very near future.

Initiated with the pioneering work of Brassard et al. (2001) on the rapid sdB pulsator PG 0014+067, precise quantitative asteroseismic studies of EC 14026 stars have been carried on seven additional targets so far: PG 1047+003 (Charpinet et al. 2003), PG 1219+534 (Charpinet et al. 2005b), Feige 48 (Charpinet et al. 2005a; Van Grootel et al. 2008a), EC 20117-4014 (Randall et al. 2006a), PG 1325+101 (Charpinet et al. 2006), PG 0911+456 (Randall et al. 2007), and BAL 090100001 (Van Grootel et al. 2008b). In all cases, the analysis succeeded in finding a consistent optimal seismic model that provides the best simultaneous match of all the observed periodicities, thus allowing the derivation of the basic structural parameters of the scrutinized star. Among these parameters, two important quantities are evaluated at interesting levels of accuracy: the total mass $\left(M_{*}\right)$ and the mass of the residual H-rich envelope $\left(M_{\mathrm{env}}\right)$. While the total mass can, in some very rare cases, be estimated by other means (see below), direct measurements of the H-rich envelope thickness is totally out of reach without asteroseismology. With current difficulties for understanding the details of $\mathrm{sdB}$ star formation, this approach should be particularly suitable for disentangling the relative importance of various evolutionary channels, notably by providing information on the mass distribution among the pulsators (see, e.g., Fontaine et al. 2006b; Charpinet et al. 2007).

It is possible to estimate the mass of a hot B subdwarf in another way, for instance in the rare occurrence where the star belongs to a short period eclipsing binary system. Indeed, in a recent work, Vučković et al. (2007) modeled the orbital behavior of PG $1336-018$ based on high sensitivity multiband $\left(u^{\prime}, g^{\prime}\right.$, $r^{\prime}$ ) photometry obtained with the camera ULTRACAM mounted on the VLT. These authors found solutions that provide the best possible fit to the orbital light curve, thus estimating important parameters of the system components, including the mass and radius of the sdB primary. Solutions were not unique, however, due in particular to the $q$-degeneracy, $q$ being the mass ratio between the two components of the system. Meanwhile, Vučković et al. (2007) retained two favored solutions corresponding to a mass for the sdB star of either $0.466 \pm 0.006 M_{\odot}$ or $0.389 \pm 0.005 M_{\odot}$.

Beyond the inherent difficulty of finding unique solutions for masses of sdB stars in this way, it is already clear that the application of the light curve technique will be strongly limited if the goal is to build up significant statistics for establishing a mass distribution. Such systems are simply too rare for systematic studies of the sort to be carried out efficiently. 
Moreover, the derived mass distribution would likely be biased as $\mathrm{sdB}$ stars in close binaries are thought to have evolved from the common envelope (CE) channel only ${ }^{1}$. On the other hand, because the presence of pulsations is independent of the binary nature of the star (hence, a seismic mass distribution will not be biased in this respect), and pulsators are much more common than close eclipsing binaries (thus allowing meaningful statistical studies), asteroseismology obviously remains the diagnostic tool with the highest potential in this domain. The major interest of PG 1336-018 resides in that the sdB primary is also an EC 14026-type pulsator, thus allowing, for the first time, to confront (and eventually calibrate) the two independent techniques. Hence, this system offers the ideal conditions to set up a new stringent test of the asteroseismic technique that we have developed for pulsating sdB stars. We point out here that another important test of our method, comparing the seismic solutions obtained with and without a priori mode identification (as can be obtained, for instance, from independent multicolor photometry or time-resolved spectroscopy), has been presented by Van Grootel et al. (2008b) in the first of this short series of papers.

In this article, we present a detailed asteroseismic analysis of the pulsating sdB star in PG 1336-018. With the help of additional spectroscopy described in Sect. 2, we propose a precise asteroseismic determination of the basic structural parameters of the pulsating sdB star, in particular its mass and radius which can both be compared with the values found by Vučković et al. (2007) from the modeling of the binary light curve (Sect. 3). In that context, we also discuss the mode identification resulting from this analysis and its implication concerning the geometrical configuration of the oscillations in this close binary system. In Sect. 4, we push the seismic analysis of PG 1336-018 into totally new grounds and present, for the first time in asteroseismology (helioseismology being the exception), a detailed inversion of the rotation profile of the pulsating $\mathrm{sdB}$ star. We also evaluate the impact on pulsation frequencies of higher-order perturbation effects induced by the fairly rapid rotation rate of the star and its tidal interaction with the companion. Finally, we provide a summary and our conclusions in Sect. 5.

\section{PG 1336-018 through the prism of spectroscopy}

\subsection{Inferred atmospheric properties from past studies}

The first estimates for the atmospheric parameters of the sdB primary in PG 1336-018 must be credited to Kilkenny et al. (1998) as part of their discovery paper. These authors performed a series of dedicated spectroscopic observations on the $1.9 \mathrm{~m}$ telescope at the Sutherland site of the SAAO using the Image Tube Spectrograph. These observations were primarily aimed at monitoring the radial velocity variations of the system, but summing up individual spectrograms allowed them to construct two timeaveraged spectra suitable for atmospheric analysis. From their $\sim 1$ A resolution spectra, fitting the available Balmer line profiles with pure hydrogen LTE model atmospheres led them to derive $T_{\text {eff }}=33139 \pm 1000 \mathrm{~K}$ and $\log g=5.78 \pm 0.10$ from their first spectrum (using the $\mathrm{H}_{\gamma}$ to $\mathrm{H}_{9}$ lines) and $T_{\text {eff }}=32895 \pm 1000 \mathrm{~K}$ and $\log g=5.67 \pm 0.10$ from their second spectrum (using the $\mathrm{H}_{\delta}$ to $\mathrm{H}_{9}$ lines). Hence, averaging these values, the atmospheric parameters of PG 1336-018 can be estimated to be

\footnotetext{
1 Although we point out that such a suspected bias can also be seen as an interesting opportunity to single out one channel among all others and study the end product of it.
}

$T_{\text {eff }}=33000 \pm 1000 \mathrm{~K}$ and $\log g=5.73 \pm 0.10$. Kilkenny et al. (1998) also examined ultraviolet spectrophotometric data available for PG 1336-018 in the IUE Final Archive. By computing the energy distribution in the 1150-5500 $\AA$ range for various effective temperatures (assuming $\log g=6.0$ for the star) and interstellar reddening values, they found a best-fit solution to the observed spectrum in that wavelength range for $T_{\text {eff }}=33000 \pm 3000 \mathrm{~K}$ and $E(B-V)=0.05$. This independent estimate of $T_{\text {eff }}$, although less accurate, confirms the value derived from the optical spectra.

More spectroscopy of PG 1336-018 was recently presented by Vučković et al. (2007). In the context of that work, the main goal was to acquire new time-resolved spectroscopy of PG 1336-018 to produce a radial velocity curve with a good phase coverage of the orbital motion. This was done using the high-resolution Ultraviolet Visual Echelle Spectrograph (UVES) during one full night obtained on the VLT at the Paranal Observatory in Chile. By co-adding all Echelle spectra taken during these observations, the authors also produced a very high signal-to-noise ratio, high resolution $(R \sim 46890)$ spectrum covering the range 3900-5000 $\AA$. However, this high- $S / N$ spectrum is plagued with discontinuities and distortions resulting from imperfect merging of the orders in the Echelle spectrogram. Meanwhile, by attempting to fit $\mathrm{H} / \mathrm{He}$, LTE models from Heber et al. (2000) to the available Balmer lines (minus $\mathrm{H}_{\gamma}$, obviously polluted by a large discontinuity in its red wing; see their Fig. 5) and He lines in this spectrum, Vučković et al. (2007) obtained the following estimates for the atmospheric parameters of the sdB primary: $T_{\mathrm{eff}}=31300 \pm 250 \mathrm{~K}, \log g=5.60 \pm 0.05$, and $\log N(\mathrm{He}) / N(\mathrm{H})=-2.93 \pm 0.05$. The values for the effective temperature and surface gravity are somewhat lower than those measured by Kilkenny et al. (1998). However, the authors recognize that the Echelle extraction problem encountered with this spectrum is likely to affect the results from the model atmosphere fit, especially for the evaluation of $\log g$, a parameter particularly sensitive to the wing shape of the Balmer lines. They also stress that "such a low $\log g$ is inconsistent with any realistic mass-radius relationship that can be derived from the orbit by at least 0.15 dex", thus advising caution about the atmospheric parameters they provide.

\subsection{New evaluation from high-S/N, low-resolution spectra}

Considering the key role that spectroscopic constraints usually play in discriminating between various possible asteroseismic solutions (see, e.g., Charpinet et al. 2005b,a, for examples of this), we found it necessary, in the situation summarized above, to obtain additional measurements for the atmospheric parameters of the sdB primary. To this end, we first analyzed an optical spectrum of PG 1336-018 with $\sim 6 \AA$-resolution and $S / N \sim 80$ covering the range from $3060 \AA$ to $5240 \AA$ kindly obtained for us by Pierre Bergeron at the Steward Observatory $2.3 \mathrm{~m}$ Telescope. We will refer to this new spectrum as PB6. We also obtained at the same telescope another spectrum, with a lower resolution ( $~ \AA$ A ) but higher sensitivity $(S / N \sim 175)$, covering the range from $3620 \AA$ to $6900 \AA$. We will refer to that one as BG9.

We analyzed these new time-averaged spectra of PG 1336-018 with our grid of NLTE, H/HE model atmospheres specifically designed for subdwarf B stars. These banks of atmosphere models and synthetic spectra were computed recently with the help of the public codes TLusty and Synspec (Hubeny \& Lanz 1995; Lanz \& Hubeny 1995). Some properties of these models are discussed in Charpinet et al. (2005b), 

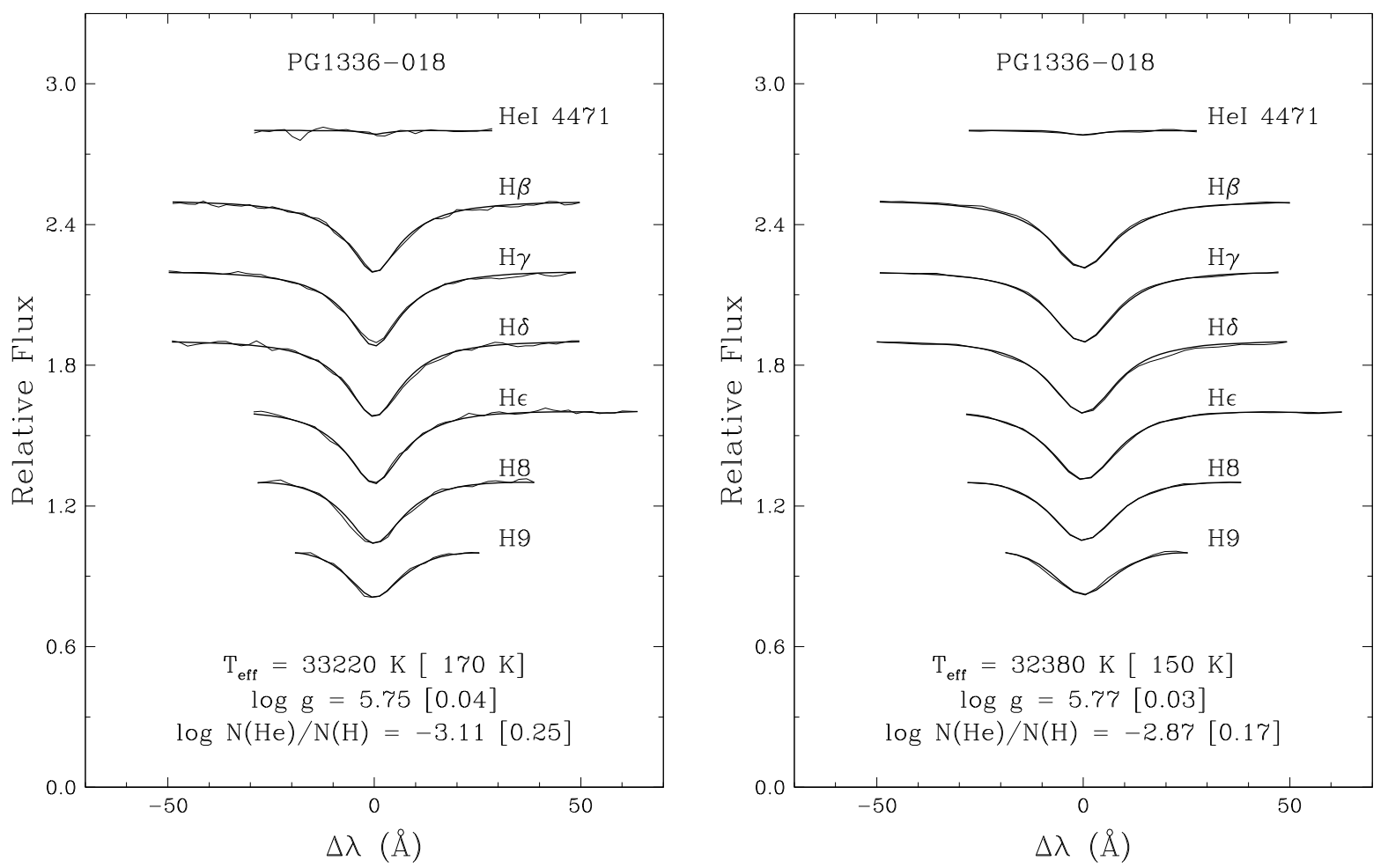

Fig. 1. Model fits (thick curves) to the hydrogen and helium lines (thin curves) available in our time-averaged high signal-to-noise, low-resolution spectra PB6 (left panel) and BG9 (right panel).

and further details will be provided in Green et al. (2008, in preparation). The best-fit results for these two spectra are shown in Fig. 1. In both cases, the line profiles are very well reproduced for the best model atmospheric parameters given in each panel. Note that the quoted uncertainties are the formal errors of the fit and do not include external errors. These two estimates, evaluated from independent spectra, show a remarkable internal consistency and, moreover, are in close agreement with the evaluation of Kilkenny et al. (1998). In this respect, our spectroscopic determinations confirm the earliest values derived by these authors, as opposed to the more recent measurement provided by Vučković et al. (2007). The clarification of this particular issue constitutes an important step for the success of the detailed asteroseismic study that follows. Note that the value given for the atmospheric He abundance by Vučković et al. (2007) is consistent with ours, as one may expect since the relatively narrow helium lines do not suffer much from the order extraction problem encountered by these authors with their Echelle spectrum.

In summary, combining the results obtained from our two low-resolution spectra, we propose the weighted mean values for the atmospheric parameters of PG 1336-018: $T_{\text {eff }}=32780 \pm 200 \mathrm{~K}, \log g=5.76 \pm 0.03$, and $\log N(\mathrm{He}) / N(\mathrm{H})=-2.94 \pm 0.14$. These values will be our favored guides in the following attempt to isolate a unique asteroseismic solution for this star.

\section{PG 1336-018 in the light of asteroseismology}

\subsection{The pulsation periods}

After the discovery of Kilkenny et al. (1998) that established both the pulsating and eclipsing binary nature of PG 1336-018, this system became the target of several follow-up multisite observations, including a fully dedicated Whole Earth Telescope (WET) photometric campaign (codename: Xcov17). Held in
April 1999 for its main part, the Xcov17 campaign resulted in $\sim 172$ h of observations for a coverage of $\sim 47 \%$, which still constitutes the most extensive monitoring available to date for PG 1336-018. The detailed analysis of the Xcov17 light curves published by Kilkenny et al. (2003) revealed that the oscillating sdB primary has a rich pulsation spectrum, thus positioning this star as a target of choice for asteroseismology.

The present seismic analysis relies on the pulsation periods uncovered in the above mentioned work. We have selected 25 out of the 28 periodicities listed in Table 4 of Kilkenny et al. (2003). These, for convenience, are reproduced in Table 1, where the identification number $\left(f_{\mathrm{n}}\right)$ given to each period follows the convention adopted by Kilkenny et al. (2003), who ranked the periods by order of decreasing amplitude. The three periods left aside $\left(f_{10}, f_{13}\right.$, and $\left.f_{8}\right)$ are all very close - i.e., of the order of the campaign frequency resolution - to peaks of higher amplitudes in the Fourier spectrum $\left(f_{5}, f_{2}\right.$, and $f_{1}$, respectively). Backing up the comments of Kilkenny et al. on this, we agree that these periods could be real pulsation modes, but they may as well originate from apparent amplitude variations due, for instance, to instrumental differences and/or heterogeneous observing conditions that can easily occur in such a large multisite campaign. Hence, we decided to simply ignore these three periods for the present asteroseismic analysis in order to avoid any risk of overly constraining the pulsation models and biasing the solution with possible spurious modes. We stress that the 25 remaining periods easily provide enough modes to tightly constrain the asteroseismic solution. In this context, we also point out that all quantitative asteroseismic studies of EC 14026 pulsators conducted so far relied on significantly less observed periods.

\subsection{On the nature of the problem}

While well suited at first sight for asteroseismology because of its rich period spectrum, PG 1336-018 has remained a 
Table 1. Periods and amplitudes of 25 modes found by Kilkenny et al. (2003) and selected for asteroseismology.

\begin{tabular}{lccl}
\hline \hline Id. & $\begin{array}{c}\text { Period } \\
(s)\end{array}$ & $\begin{array}{c}\text { Amplitude } \\
(\%)\end{array}$ & Comments \\
\hline$f_{18}$ & 204.70 & 0.07 & full campaign only \\
$f_{27}$ & 195.65 & 0.05 & \\
$f_{21}$ & 191.61 & 0.06 & \\
$f_{16}$ & 186.69 & 0.08 & \\
$f_{3}$ & 186.24 & 0.37 & \\
$f_{4}$ & 185.45 & 0.25 & \\
$f_{25}$ & 185.15 & 0.06 & \\
$f_{5}$ & 183.98 & 0.20 & period $\left(f_{10}\right)$ at 184.05 s ignored \\
$f_{7}$ & 183.68 & 0.17 & \\
$f_{22}$ & 182.79 & 0.06 & \\
$f_{17}$ & 181.63 & 0.08 & \\
$f_{12}$ & 181.27 & 0.10 & \\
$f_{2}$ & 179.03 & 0.40 & period $\left(f_{13}\right)$ at 178.96 s ignored \\
$f_{6}$ & 178.62 & 0.17 & full campaign only \\
$f_{20}$ & 177.90 & 0.07 & period $\left(f_{8}\right)$ at 173.59 s ignored \\
$f_{1}$ & 173.69 & 0.47 & \\
$f_{15}$ & 169.74 & 0.09 & \\
$f_{11}$ & 169.03 & 0.10 & \\
$f_{28}$ & 162.25 & 0.05 & full campaign only \\
$f_{9}$ & 141.42 & 0.13 & \\
$f_{14}$ & 140.67 & 0.09 & \\
$f_{23}$ & 134.90 & 0.06 & full campaign only \\
$f_{19}$ & 126.89 & 0.07 & \\
$f_{24}$ & 125.81 & 0.06 & \\
$f_{26}$ & 96.95 & 0.50 & \\
\hline & & & \\
& &
\end{tabular}

challenging object due to its close binary nature. Indeed, tidal interaction in this system is strongly expected to lead to orbit circularization and spin-orbit synchronization between the two stellar components. This hypothesis will be investigated further in the following seismic analysis, but this implies that the sdB pulsator likely rotates as a solid body with a period $P_{\text {rot }}=2.42438 \mathrm{~h}$, identical to the orbital period of PG 1336-018 accurately measured by Kilkenny et al. (2000). This would mean that the pulsating sdB star is a fairly fast rotator, thus generating complications for the asteroseismic analysis that we detail below.

In standard pulsation theory (see, e.g., Unno et al. 1989), oscillation eigenmodes are characterized by three quantum numbers, respectively, the radial order $k$, the degree $\ell$, and the azimuthal order $m$. The two last indices are associated with the spherical harmonics $Y_{l}^{m}(\theta, \phi)$ that represent the nonradial (angular) geometrical aspect of the oscillation modes, while $k$ is the number of nodes of the eigenfunction in the radial direction between the surface and the center of the star. The main effect of slow stellar rotation on pulsations is to lift, for nonradial $(\ell \geq 1)$ modes, the $m$ degeneracy that exists on frequencies (or periods) when the star is considered spherically symmetric, i.e., nonrotating $^{2}$. This leads to the splitting of all $\{\ell, k, m=0\}$ modes of frequency $v_{k \ell 0}$ into their $2 \ell+1$ components (differing by their $m$ values, with $-\ell \leq m \leq+\ell$ ), each now having a different frequency $v_{\mathrm{klm}}$ (here, and from now on, we use by convention $v=1 / P$, where $P$ is the period the mode). Generally treated as a perturbation, rotational splitting, at the first order of approximation (valid for a slowly rotating star, see below), creates groups

\footnotetext{
${ }^{2}$ Of course, all stars must rotate. The assumption of no rotation is a common simplification used in stellar and pulsation modeling, which is well justified as long as rotation does not affect significantly the stellar structure itself. In this situation, rotation can be considered as a small perturbation to the equilibrium structure of a nonrotating star.
}

of evenly spaced multiplets in the frequency domain ${ }^{3}$. In the case of solid body rotation relevant for a star like PG 1336-018, the multiplet components are separated by

$\Delta v_{k \ell}=V_{\text {rot }}\left(1-C_{k \ell}\right)$,

and their frequencies are given by the relationship

$v_{k \ell m}=v_{k \ell 0}-m \Delta v_{k \ell}$.

Here, $V_{\text {rot }} \equiv \Omega_{\text {rot }} / 2 \pi=1 / P_{\text {rot }}$ is the rotation rate of the star (in $\mathrm{Hz}$ ) and $C_{k \ell}$, the dimensionless rotation coefficient (also known as the Ledoux coefficient; Ledoux 1951), specific to each mode of radial order $k$ and degree $\ell$, is defined as

$C_{k \ell}=\frac{\int_{0}^{R}\left\{\xi_{h}^{2}+2 \xi_{r} \xi_{h}\right\} \rho r^{2} \mathrm{~d} r}{\int_{0}^{R}\left\{\xi_{r}^{2}+\ell(\ell+1) \xi_{h}^{2}\right\} \rho r^{2} \mathrm{~d} r}$,

with $\xi_{r}(r)$ and $\xi_{h}(r)$ being respectively the zeroth-order (i.e., nonperturbed) radial and horizontal displacement eigenfunctions of the mode.

For PG 1336-018 in which the stellar components are most likely locked tidally to the orbital motion, the expected frequency spacing due to rotational splitting is approximately $\Delta v \sim 1 / P_{\text {rot }} \sim 114 \mu \mathrm{Hz}$. This value, roughly estimated by simply neglecting the $C_{k \ell}$ coefficients which are typically small $(\lessgtr 0.10)$ for $p$-modes, is sufficiently large, especially viewed as a factor of the $m$ index in Eq. (2), compared to the typical frequency spacings between modes of adjacent $k$ and/or $\ell$ indices (see, e.g., Table 3) that several multiplets are bound to overlap in the frequency domain. This constitutes a serious difficulty for identifying a priori the central $m=0$ components of multiplets. So far, this a priori identification has been a prerequisite in all our previous seismic analyses of slowly rotating pulsators. An eventual misidentification of the $m=0$ component for a slowly rotating star where rotational splitting generates multiplets of frequencies separated only by a few $\mu \mathrm{Hz}$ has, in practice, no significant impact on the determination of the seismic solution (see, e.g., Van Grootel et al. 2008b). Obviously, this cannot be true for a moderate rotator like PG $1336-018$, where an error of $\sim 114 \mu \mathrm{Hz}$ on one or several modes due to such eventual misidentifications would certainly impact considerably the asteroseismic solution. The situation is even worse when we consider the mode dependent $C_{\mathrm{k} \ell}$ contribution to the frequency spacings. Although the values of this coefficient are generally small for $p$-modes, they can still contribute to as much as $\sim 10 \%$ of the rotational splitting. Hence, the multiplets are likely to have their own values of $\Delta v$ with differences reaching as much as $\sim 11 \mu \mathrm{Hz}$ approximately in the $103-114 \mu \mathrm{Hz}$ range. This complicates significantly the task of identifying multiplet components, as rotational splitting no longer produce the same frequency spacing for all multiplets. To add to the difficulty of making a sensible a priori identification, and basically render the task nearly impossible, higher-order perturbation effects may become significant when stellar rotation reaches moderate rates like the one observed in PG 1336-018 (see Sect. 4.2). These effects add a nonuniform contribution to the frequency for each component in a given multiplet. In other words, the symmetry of each multiplet (i.e., the equal frequency spacings) is no longer preserved and the relatively clear signature of rotation simply disappears.

The only objective approach allowing us to bypass the serious difficulties explained above is to fully integrate stellar rotation and its effects on the pulsation frequencies in the detailed

\footnotetext{
3 Note that even frequency spacings only occur if the rotation is spherically symmetric, as we assume in this study.
} 

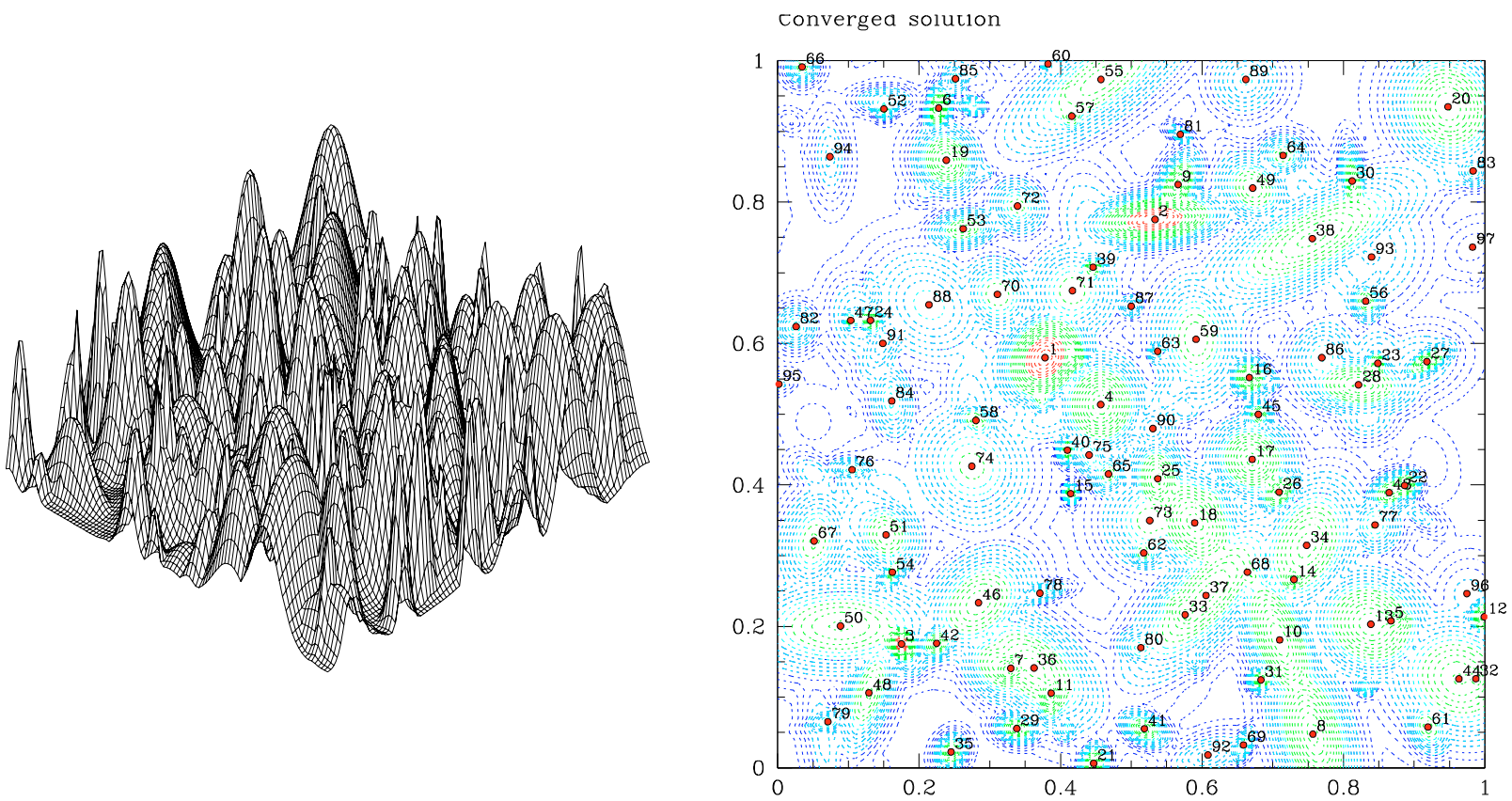

Fig. 2. Capabilities of the multimodal hybrid GA optimization code to find all relevant optima (here maxima) of a complex function. The test function is, for clarity and illustration purposes, a 2-dimensional landscape built with 200 random gaussians, except for the three highest peaks whose respective height and position in parameter space are fixed. Left (right) panel is a 3D (contour) representation of the function. In the right panel, each numbered filled circle shows where a solution is found by the code. The number corresponds to the rank of the solution, in order of decreasing height. This result was obtained using the GA code with a population of 500 individuals evolved over 100 generations.

asteroseismic analysis. When no longer using purely spherical pulsation models, the need for identifying a priori - and sometimes subjectively - the central $(m=0)$ components of multiplets is suppressed. This way, the whole period spectrum generated by the $\{k, \ell, m\}$ eigenmodes (and not only by the $\{k, \ell, m=0\}$ modes, in the purely spherical case) can be used in the seismic fitting procedure. This was done and tested for the first time by Van Grootel et al. (2008a) in a re-analysis of the moderately rotating pulsating sdB star Feige 48. We describe this modified approach adapted to the case of PG 1336-018 in the next subsection.

\subsection{Strategy and tools for a challenging pulsating star}

The method and tools developed to perform objective asteroseismology of sdB pulsators have been described in some details in Charpinet et al. (2005b, and references therein). In a nutshell, the method follows the so-called "forward modeling" strategy that consists of comparing quantitatively computed pulsation periods for large batches of stellar models of varying parameters to the observed period spectrum of the scrutinized star. By optimizing this comparison to find the best possible match (or matches) to the observations, one seeks to determine precisely some of the inner properties and parameters of the star. Two software packages exist to achieve this goal, one developed in Montréal and the other in Toulouse which mainly differ at the level of the numerical codes and algorithms used to explore the model parameter space. Needless to mention that both packages, although using different optimization techniques, have always led to identical results, thus providing an excellent guarantee that the numerical tools themselves are robust and reliable.

In the present analysis of PG 1336-018, the Toulouse package is used. It is designed to efficiently perform a double optimization procedure as described in Charpinet et al. (2005b), using codes based on Genetic Algorithm (GA) implementations. The double optimization scheme consists of finding simultaneously the best association of observed/computed periods along with the best fitting model parameters. In this procedure, the quality of the fit is evaluated quantitatively through a merit function, $S^{2}$, defined as

$S^{2}\left(a_{1}, a_{2}, \ldots, a_{N}\right)=\sum_{i=1}^{N_{\mathrm{obs}}}\left(\frac{P_{\mathrm{obs}}^{(i)}-P_{\mathrm{th}}^{(i)}}{\sigma_{i}}\right)^{2}$,

where $N_{\text {obs }}$ is the number of observed periods, $\left\{a_{1}, a_{2}, \ldots, a_{N}\right\}$ are the model parameters (in the general case), and each $\left\{P_{\mathrm{obs}}^{(i)}, P_{\mathrm{th}}^{(i)}\right\}$ is an associated pair of observed/theoretical periods for that model. Two minimizations are required because, without strict a priori mode identification (which is normally not available), the best possible association between observed and computed periods for a given model (a first minimization of $S^{2}$ viewed as a combinatorial optimization problem) must be found at the same time as $S^{2}\left(a_{1}, a_{2}, \ldots, a_{N}\right)$ is minimized in the $N$-dimensional model parameter space (i.e., the second optimization). Our numerical tools to achieve this include a GA-based period matching algorithm, for the first combinatorial minimization, and a massively parallel hybrid-GA code for multimodal optimization of functions in $\mathrm{N}$-dimension to explore efficiently the model parameter space in search of the best-fit solution (the global minimum of the $S^{2}$ function) and all relevant secondary solutions (i.e., eventual local minima of $S^{2}$ that may also be of interest).

A detailed description of the latter code is beyond the scope of this paper and shall be presented elsewhere, but for illustrative purposes, Fig. 2 demonstrates its capabilities to detect all significant optima (maxima in that case) of a complex test function (see details provided in the figure caption). With this representative example, we simply emphasize the robustness of this 
code in finding the best optimum, as well as all relevant secondary optima of a given function, even in difficult situations. Hence, when used in the context of asteroseismology to localize minima of the $S^{2}\left(a_{1}, a_{2}, \ldots, a_{N}\right)$ function, this code ensures with a high level of confidence that the best solution (of lowest $S^{2}$ value) is indeed recovered, as well as all potentially interesting secondary solutions (of higher $S^{2}$ values). Therefore, the code provides two very important guarantees regarding the objectivity of the method: first, a complete and exhaustive exploration of the entire model parameter space is done and second, feedback on the uniqueness (or not) of the solution is available.

The evaluation of $S^{2}$ requires the computation of theoretical period spectra to compare with the observed periods. This procedure involves several steps. First, the structure of a subdwarf B star of given parameters (see below) is computed. As in previous seismic analysis done so far, the structure is obtained with our "second generation" models appropriate for sdB asteroseismology. We recall that these models are static structures expanding as deep as $\log q \equiv \log \left(1-M(r) / M_{*}\right) \simeq-0.05$ that incorporate nonuniform abundance profiles of iron derived from detailed microscopic diffusion calculations assuming an equilibrium between gravitational settling and radiative levitation. The latter is a key ingredient to construct reliable models of pulsating $\mathrm{sdB}$ stars (see, e.g., Fontaine et al. 2006a). Four fundamental parameters are needed to fully specify the structure of an $\mathrm{sdB}$ star with these models: the effective temperature $T_{\text {eff }}$, the surface gravity $\log g$, the total mass of the star $M_{*}$, and the logarithmic fractional mass of the H-rich envelope $\log q(H) \equiv \log \left[M(H) / M_{*}\right]$. The second step is the evaluation of the adiabatic pulsation properties of the model, assumed purely spherical at this stage, using an efficient and robust code based on finite element techniques (Brassard et al. 1992; Brassard \& Charpinet 2008). The novelty in the present analysis is to incorporate a third step to account for the effects of stellar rotation on the pulsation spectrum. This is done by computing for each mode the first-order perturbations of the pulsation frequencies caused by rotation according to Eqs. (1)-(3) given in Sect. 3.2. Note that, in this procedure, we explicitly neglect higher-order perturbation effects (but see Sect. 4.2) and we assume solid body rotation, a choice a priori justified by the fact that PG $1336-018$ is very likely tidally locked. We later discuss, in light of our best-fit solution, the relevance of this hypothesis and explore possible departures from it (see Sect. 4.1). With this complete rotationally-split theoretical period spectrum available (each mode now being nondegenerate and uniquely identified by its $k, \ell$, and $m$ indices), the first optimization step mentioned above is performed by the period matching code.

\subsection{Optimal solution in the model parameter space}

The search for best-fit solutions to the observed periods of PG 1336-018 was launched in a vast parameter space domain defined as follows: $30000 \mathrm{~K} \leq T_{\text {eff }} \leq 36000 \mathrm{~K}, 5.6 \leq \log g \leq$ $5.9,-5.2 \leq \log q(H) \leq-2.0$, and $0.3 \leq M_{*} / M_{\odot} \leq 0.7$. These quantities are the four natural parameters that define the $\mathrm{sdB}$ star model, as mentioned previously. With the inclusion of rotational splitting in the fitting procedure, there is in the context of the solid body rotation law assumed here, a fifth parameter, $P_{\text {rot }}$, which specifies the rotation period of the star and controls the magnitude of the frequency splitting through Eq. (1). While $P_{\text {rot }}$ could also be considered as a free parameter in our initial search, we chose instead to fix it at the same value as the orbital period of the binary in order to be fully consistent with the initial hypothesis that the system has reached full spin-orbit synchronism and rotates as a solid body. Hence, we used $P_{\text {rot }}=P_{\text {orb }}=2.42438 \mathrm{~h}$ (or $\left.8727.78 \mathrm{~s}\right)$, as measured by Kilkenny et al. (2000). The ranges for the four structural parameters are set according to various constraints: the limits on $T_{\text {eff }}$ and $\log g$ are loosely based on the independent spectroscopic estimates of these atmospheric parameters, whereas the ranges of $\log q(H)$ and $M_{*}$ rely on stellar evolution constraints, including various possible formation scenarios (see, Han et al. 2002, 2003).

For the period computation part, we considered all modes of degree $\ell=0,1,2$, and 4 in the 50-500 s period range, thus covering amply the range observed in PG 1336-018 (see Table 1). The upper limit for the degree $\ell$ corresponds to the minimum value that can account for the mode density in the observed period range. Indeed, a limit of $\ell \leq 2-$ often assumed in asteroseismic studies based on general visibility arguments does not provide enough theoretical modes to allow reasonable fits to the period distribution observed in PG 1336-018 and, therefore, must clearly be excluded on this basis. This fact, again, constitutes a strong indication that modes of degree $\ell>2$ must be observable in EC 14026 stars, and that rotational splitting, which is already included in the present analysis, cannot be an alternative to account for such a mode density. Note that we explicitly excluded the $\ell=3$ modes, following recent results indicating that these modes are less visible (in the optical domain) than the $\ell=4$ modes in sdB stars, due to cancellation effects (Randall et al. 2005a; Tremblay et al. 2006; Randall et al. 2007). We cannot formally rule out the presence of $\ell=3$ (or even $\ell>4$ ) modes in the observed period spectrum of PG 1336-018, but our approach explicitly excludes this possibility at this stage. Nevertheless, despite this uncertainty, we stress that with the limitations we impose on the degree $\ell$ of the modes, the observed period spectrum can fully be explained, as shown below. We interpret this as an indication that our assumptions at this specific level are reasonable. Finally, we mention that, as in previous studies (see, e.g., Brassard et al. 2001), we used the inverse of the theoretical mode density of each model as a global weight in the evaluation of $S^{2}$ : in Eq. (4) we set $\sigma_{i}=\sigma_{\mathrm{d}}$, where $\sigma_{\mathrm{d}}$ is the ratio of the width of the considered period window (here $450 \mathrm{~s}$ ) to the number of modes in that window. This technical choice does not affect the localisation of the solutions but partly removes eventual biases toward unrealistic models having significantly higher mode densities than the observed spectrum.

Within the four-dimensional search domain specified, the optimization code spotted several regions that provide similarly good matches (i.e., within 1- $\sigma$ of the best-fit solution as measured by the quantity $S^{2}$ ) to the 25 periods observed in PG 1336-018. The multiplicity of solutions is typical of sdB asteroseismology and it usually requires the use of additional constraints or considerations to lift the ambiguities. Not surprisingly, a more complex case such as PG 1336-018 does not transgress this rule. Among the possible solutions, however, one in particular has retained our attention for its outstanding internal consistency as compared to the other families of potentially good models. We further discuss below the properties of this optimal solution.

Figure 3 shows maps of the $S^{2}$ function (given on a logarithmic scale) around the optimal model solution that has attracted our attention. The location of the optimal model in these maps is indicated by a yellow mark and its structural parameters are $T_{\text {eff }}=32743 \mathrm{~K}, \log g=5.7391, \log q(H)=-4.6336$, and $M_{*}=0.4585 M_{\odot}$. This model solution, with a $S^{2}$ value of 1.89 $\left(\log S^{2}=0.28\right)$, offers an excellent match to the 25 observed periods considered in this asteroseismic analysis. We discuss 

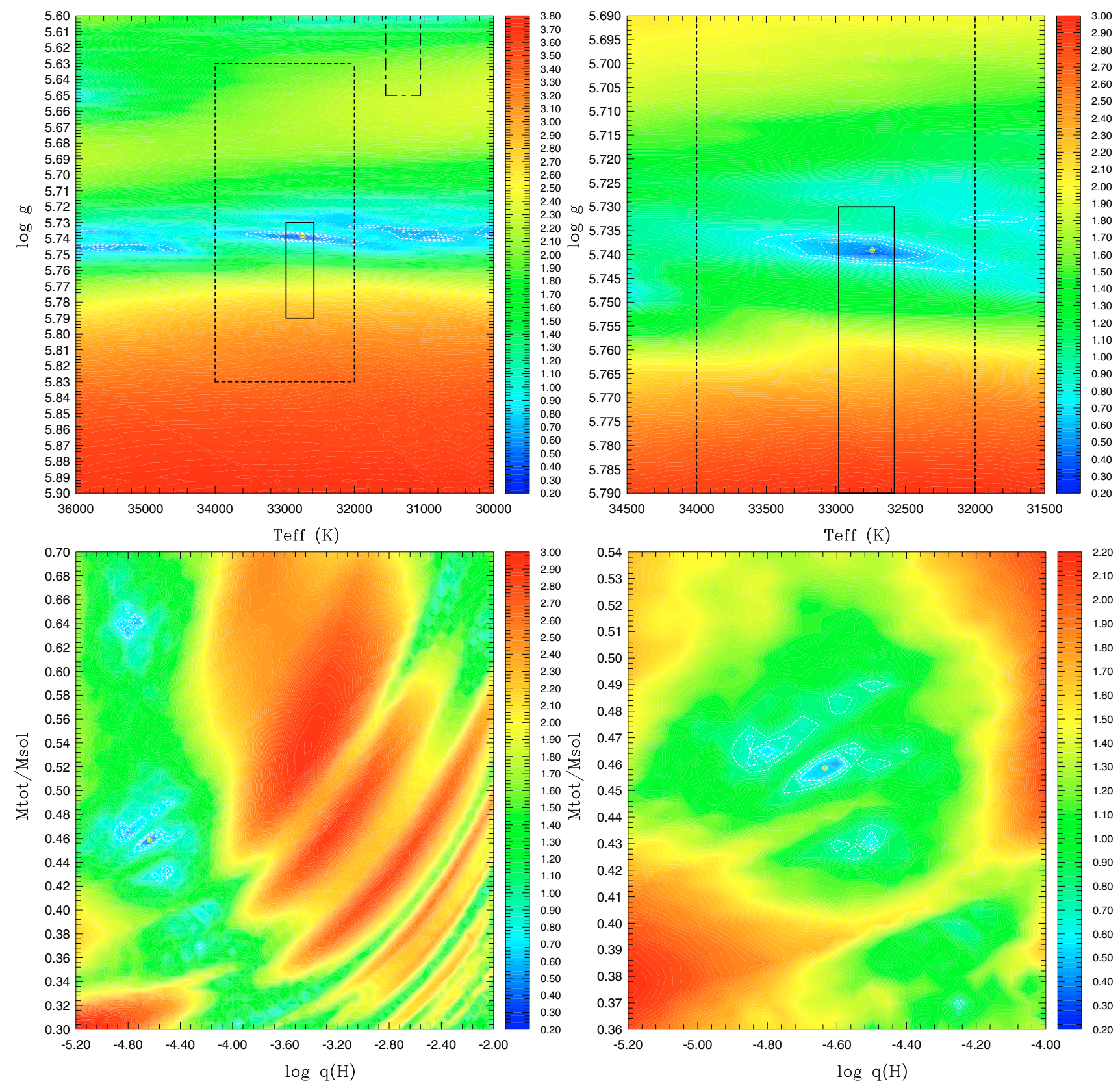

Fig. 3. $S^{2}$ maps (on a logarithmic scale) around the optimal model solution indicated by a yellow mark. Right panels are close-up views of the solutions shown in the corresponding left panels. The upper plots represent slices of the 4-dimensional $S^{2}$ function in the $\log g-T_{\text {eff }}$ plane, while the lower plots are slices along the $\log q(H)-M_{*}$ plane. All slices are centered on the optimal solution. White contours show regions where the period fits have $S^{2}$ values within, respectively, the 1- $\sigma, 2-\sigma$, and 3- $\sigma$ confidence level relative to the best-fit solution. The solid-line, dotted-line, and dashed-dotted-line rectangles show, respectively, the spectroscopic constraints derived from the analysis of our spectra, from Kilkenny et al. (1998), and from Vučković et al. (2007). In the later case, we included these estimates for completeness sake, but, as discussed in Sect. 2.1, there are known problems associated with this measurement.

below, in Sect. 3.6, the details of this match and the corresponding mode identification that is derived. The first striking property of this model solution is its remarkable consistency with the independent measurements of the atmospheric parameters $\log g$ and $T_{\text {eff }}$ derived from spectroscopy. These are represented as rectangles in the upper panels of Fig. 3 (see figure caption for details). For completeness, we included the measurements of Vučković et al. (2007) in the plots, but, for the reasons given in Sect. 2 above, we ignore in what follows that outlying spectroscopic box. Apart from that, we find that the agreement is particularly good with our own independent spectroscopic measurements, and that of Kilkenny et al. (1998).
The consistency between the spectroscopic and asteroseismic solutions is one important factor that has contributed to prefer this model over other solutions found to have comparable $S^{2}$ values in our exhaustive exploration of the model parameter space. A closer look at the upper-left panel of Fig. 3 shows, indeed, that several other regions of good-matching models (i.e., within the 1-, 2-, or 3- $\sigma$ limits relative to the lowest $S^{2}$ value represented as white dashed-line contours) do exist. However, most of them (those revealed in Fig. 3 and a few others in regions of the model parameter space not represented here) can be rejected on the basis of our stringent spectroscopic constraints. This proves, once again, that accurate spectroscopy is 
Table 2. Structural parameters of PG 1336-018 ( $y=13.450 \pm 0.093$; Wesemael et al. 1992) derived from asteroseismology, spectroscopy, and compared with the results from the independent analysis of the orbital light curve proposed by Vučković et al. (2007).

\begin{tabular}{lcccccc}
\hline \hline \multicolumn{2}{c}{ Asteroseismic analysis } & \multicolumn{2}{c}{ Spectroscopy \& misc. } & \multicolumn{2}{c}{ Vučković et al. (2007) } \\
\hline Quantity & Estimated value & This study & $\begin{array}{c}\text { Kilkenny et al. } \\
\text { (1998) }\end{array}$ & Model I & Model II & Model III $^{\ddagger}$ \\
\hline$T_{\text {eff }}(\mathrm{K})$ & $32740 \pm 400$ & $32780 \pm 200$ & $33000 \pm 1000$ & $\ldots$ & $\ldots$ & $\ldots$ \\
$\log g$ & $5.739 \pm 0.002$ & $5.76 \pm 0.03$ & $5.73 \pm 0.1$ & $5.74 \pm 0.05$ & $5.77 \pm 0.06$ & $5.79 \pm 0.07$ \\
$M_{*} / M_{\odot}$ & $0.459 \pm 0.005$ & $\ldots$ & $\ldots$ & $0.389 \pm 0.005$ & $0.466 \pm 0.006$ & $0.530 \pm 0.007$ \\
$\log \left(M_{\text {env }} / M_{*}\right)$ & $-4.54 \pm 0.07$ & $\ldots$ & $\ldots$ & $\ldots$ & $\ldots$ & $\ldots$ \\
$R_{*} / R_{\odot}\left(M_{*}, g\right)$ & $0.151 \pm 0.001$ & $\ldots$ & $\ldots$ & $0.14 \pm 0.01$ & $0.15 \pm 0.01$ & $0.15 \pm 0.01$ \\
$L_{*} / L_{\odot}\left(T_{\text {eff }}, R\right)$ & $23.3 \pm 1.5$ & $\ldots$ & $\ldots$ & $\ldots$ & $\ldots$ & $\ldots$ \\
$M_{V}\left(g, T_{\text {eff }}, M_{*}\right)$ & $4.49 \pm 0.04$ & $\ldots$ & $4.1 \pm 0.2$ & $\ldots$ & $\ldots$ & $\ldots$ \\
$d\left(V, M_{V}\right)(\mathrm{pc})$ & $619 \pm 38$ & $\ldots$ & $710 \pm 50$ & $\ldots$ & $\ldots$ & $\ldots$ \\
$\log N(\mathrm{He}) / N(\mathrm{H})$ & $\ldots$ & $-2.94 \pm 0.14$ & $\ldots$ & $\ldots$ & $\ldots$ & $\ldots$ \\
$P_{\text {rot }}(\mathrm{h})^{\dagger}$ & 2.42438 & $\ldots$ & $\ldots$ & $\ldots$ & $\ldots$ \\
$V_{\mathrm{eq}}\left(P_{\mathrm{rot}}, R\right)\left(\mathrm{km} \mathrm{s}^{-1}\right)$ & $75.9 \pm 0.6^{*}$ & $\ldots$ & $\ldots$ & $\ldots$ & $\ldots$ \\
\hline
\end{tabular}

$\dagger$ Assumed identical to the orbital period (value taken from the ephemeris of Kilkenny et al. 2000). $\doteqdot$ Deemed unlikely and, thus, rejected by Vučković et al. (2007) due to the high mass of the primary. ${ }^{*}$ Hence, we predict that $V \sin i=74.9 \pm 0.6 \mathrm{~km} \mathrm{~s}^{-1}$ for the rotating sdB star $\left(i \sim 81^{\circ}\right)$, a value that can be tested from spectroscopy.

often essential in the process of finding unique asteroseismic solutions. This is particularly important in the present analysis since the shape of $S^{2}$ function turns out to be significantly more complex than it was in our previous asteroseismic studies. The latter is a consequence of the introduction of rotationallysplit period spectra in the optimization procedure. With 25 periods to work with, a number significantly larger than in our previous experiments, the number of combinations available to match theoretical modes with the observed periods largely increases. Further problems linked to this increased complexity are illustrated in the lower panels of Fig. 3. These represent the $\log q(H)-M_{*}$ plane at fixed $T_{\text {eff }}$ and $\log g$ values (set to the values of the optimal model). The region of lowest $S^{2}$ values in which our preferred solution lies, near $M \sim 0.46 M_{\odot}$ and $\log q(H) \sim-4.6$, clearly appears in these maps, but several areas with $S^{2}$ values within the 3-, 2-, and sometimes 1- $\sigma$ contours are also present, notably near $M \sim 0.64,0.47,0.43$, and $0.36 M_{\odot}$. These models cannot be rejected on the basis of spectroscopy alone because they remain consistent with it and, furthermore, they cannot be segregated either on the basis of their (comparable) values of $S^{2}$. However, a closer look at the respective solutions spotted in these areas reveals that none of these alternative models is fully satisfactory at the level of the mode identification itself, a domain where our optimal solution also features remarkable internal consistency, as we will discuss in detail below (Sect. 3.6).

\subsection{Derived structural parameters}

The optimal solution that we uncovered leads to the determination of important structural properties of PG 1336-018, following naturally from the fundamental parameters of the corresponding stellar model. These parameters are the surface gravity $\log g$, the effective temperature $T_{\text {eff }}$, the stellar mass $M_{*}$, and the mass of the H-rich envelope through the quantity $\log \left(M_{\text {env }} / M_{*}\right) \simeq \log q(H)$. Secondary quantities can be derived from these parameters, such as the stellar radius $R_{*}$ (as a function of $M_{*}$ and $g$ ), the luminosity $L_{*}$ (as a function of $T_{\text {eff }}$ and $R_{*}$ ), the absolute magnitude $M_{V}$ (as a function of $g, T_{\text {eff }}$ and $M_{*}$ in conjunction with the use of detailed model atmospheres), and the distance from Earth $d$ (as a function of apparent magnitude
$V$ and $M_{V}$ ). Moreover, with the rotation period, $P_{\text {rot }}$, equal to the orbital period in this assumed synchronized system, we can estimate the equatorial velocity $V_{\text {eq }}$ (as a function of $P_{\text {rot }}$ and $R_{*}$ ). The derived asteroseismic values for all these quantities are listed on the left-hand side of Table 2. The quoted 1- $\sigma$ uncertainties for the asteroseismic quantities are derived following the method described in Charpinet et al. (2005b).

Table 2 also provides the values derived for some of these parameters using independent techniques. We have already emphasized the excellent agreement that can be achieved between the spectroscopic and asteroseismic determinations of the effective temperature and surface gravity of the sdB star. This result is rather typical in the field of $\mathrm{sdB}$ asteroseismology, but is not fully satisfactory, however, in the sense that spectroscopy has to be used to guide the selection of the asteroseismic solution in the vast model parameter space. More interesting is the comparison that becomes possible with the parameters inferred from the totally independent analysis of the binary light curve performed by Vučković et al. (2007). From their analysis, these authors could not formally discriminate between three models that best reproduce the binary light curve of PG 1336-018, and therefore proposed three favored solutions for the system parameters. The relevant parameters of these models are reproduced on the righthand side of Table 2. These are the measured surface gravity, mass, and radius of the sdB primary allowing direct comparison with our asteroseismic results.

Table 2 immediately reveals that a very strong consistency exists between Model II of Vučković et al. (2007) and our optimal asteroseismic model of PG 1336-018. The determinations of the surface gravity $\log g$, and, more importantly, the stellar mass $M_{*}$, and the stellar radius $R_{*}$ all agree within their respective 1- $\sigma$ uncertainties, a most remarkable convergence between two completely different methods for evaluating the stellar parameters of the primary star. Indeed, such a close agreement is particularly outstanding as both the mass and radius of the star are determined very accurately with each method. This makes it highly unlikely that this remarkable agreement has occured by chance. Considering also that there could have been at the outset possible important systematic difference in both approaches, the results presented here are almost overwhelming. At this stage, we point out that none of the alternative seismic models that 
we found (those providing similarly good matches to the observed periods of PG $1336-018$ as measured by the merit function $S^{2}$ ) can be associated with any of the models of Vučković et al. (2007). This constitutes, a posteriori, another consistency argument in favor of the optimal seismic model that we isolated.

It seems particularly clear, from this exceptional convergence with our asteroseismic result, that Model II proposed by Vučković et al. (2007) is the correct solution and Models I and III must be rejected. We point out that these authors already suggested that Model III is rather unlikely due to the relatively high mass inferred for the primary. According to them, such a high mass would be difficult to explain in a common envelope evolutionary scenario, the formation channel that PG 1336-018 is expected to have evolved through. Instead, both Model II and our asteroseismic solution point toward a mass of $\sim 0.46 M_{\odot}$, i.e., very close to the peak of the mass distribution for sdB stars (see, Han et al. 2002, 2003). We also point out that the inferred value for $\log \left(M_{\text {env }} / M_{*}\right)$ indicates a rather thin H-rich envelope as one would expect, according to stellar evolution theory, for this relatively hot and compact sdB star.

\subsection{Period fit and mode identification}

The optimal seismic model selected for PG 1336-018 offers an excellent match to the 25 periods identified in this star. The resulting identification of the modes involved is given in Table 3. This table provides the derived distribution of the observed periods $P_{\text {obs }}$ (or frequencies $v_{\text {obs }}$ ) as they were matched to the computed modes with periods $P_{\text {th }}$ (or frequency $v_{\text {th }}$ ), radial order $k$, degree $\ell$, and azimuthal order $m$. The relative and absolute differences in period and frequency, $\Delta X / X$ (where $X$ is either $P$ or $v$; in $\%), \Delta P$ (in seconds), and $\Delta v$ (in $\mu \mathrm{Hz})$ for each pair $\left(P_{\mathrm{obs}}\right.$, $\left.P_{\text {th }}\right)$ is also provided.

The average relative dispersion between the fitted periods (frequencies) is $\overline{\Delta X / X} \simeq 0.17 \%$. On an absolute scale, this represents an average period dispersion of $\overline{\Delta P} \simeq 0.27 \mathrm{~s}$ (with a standard deviation around this mean of $\sim 0.20 \mathrm{~s}$ ) or an average frequency dispersion of $\overline{\Delta v} \simeq 10.5 \mu \mathrm{Hz}$ (with a standard deviation of $\sim 9.4 \mu \mathrm{Hz}$ ) with, for the worst cases, a difference of $\Delta P \sim 0.55 \mathrm{~s}$ and $\Delta v \sim 37.8 \mu \mathrm{Hz}$. The quality of this simultaneous fit is well within the standards that can currently be achieved in sdB asteroseismology. This is remarkable, considering that 25 periods had to be reproduced simultaneously, a number significantly larger than those involved in all analyses carried out so far. This result, not guaranteed at the outset, is a strong indication that our initial choice of imposing synchronous rigid rotation is correct. With 15 periods (out of 25) being identified as components of multiplets (see Table 3), and therefore having a very significant weight in the determination of the best fit solution, it is highly unlikely that a wrong hypothesis for the rotation law would lead to a period fit at this level of accuracy (for an optimal model with parameters consistent with the orbital solution, moreover). This fact strongly suggests that the sdB star in PG 1336-018 is indeed tidally synchronised and in rigid rotation, as it will be illustrated and discussed further, later on, in Sect. 4.1. We still point out, however, that the accuracy at which the frequencies are reproduced $(\sim 10.5 \mu \mathrm{Hz}$ on average) is still approximately one order of magnitude larger than the accuracy at which these periods are measured $(\sim 1 \mu \mathrm{Hz}$, the resolution in the Fourier domain resulting from the length of the observational campaign). Hence, there is clearly room for further improvements in the physics of the stellar structures used to model the interiors of sdB stars. This was already noted in our previous studies. Such improvements are among the goals to be sought in future asteroseismic studies of sdB pulsators.

Focusing now on the details of the mode identification, we note that the observed periods are identified with radial $(\ell=0)$ and nonradial $\ell=1,2$, and 4 acoustic modes with radial orders $k$ in the range 0-3 (except for one low-amplitude period identified with a $k=5$ mode). This is typical of EC 14026type pulsators. Complementary nonadiabatic pulsation calculation for this model also indicates that these modes are indeed predicted to be unstable due to the usual $\kappa$-mechanism that involves the $Z$-bump enhanced by local diffusive accumulations of iron. This optimal solution is therefore fully consistent at the nonadiabatic level as well, a consistency that has always been verified in all our previous asteroseismic studies performed to date. We also point out that, according to this mode identification, the observed periods are found in the low-radial order part of the theoretical band of unstable $p$-modes that, in this particular model, extends up to $k \sim 7$. This trend was already noticed in previous analyses and points toward a general property of rapid sdB pulsators that seems to preferentially excite the lowest-order modes within their unstable period range. The fact that our proposed mode identification for PG 1336-018 also conforms to this rule is another element accrediting the validity of the chosen optimal seismic model.

Another interesting property of this mode identification is that a consistent hierarchy in terms of apparent mode amplitudes is fairly well established. Due to spatial averaging effects occurring when the observed stellar disk is not resolved (which is always the case, except for the Sun), the visibility of modes tend to decrease when the number of nodal lines at the surface (i.e., the degree $\ell$ ) increases. For sdB stars, the apparent mode amplitudes should be maximum for the unaffected $\ell=0$ modes and should decrease for $\ell=1,2$ and 4 in that order, the $\ell=4$ modes, in particular, being much more affected. This simple argument should not be blindly applied to all individual modes, however, as their intrinsic amplitudes are not known a priori and possibly (likely?) differ very significantly from one mode to the other. For this reason, modes of higher degree $\ell$ excited with high intrinsic amplitudes can possibly show higher apparent amplitudes than modes of lower degrees but carrying less energy, thus having lower intrinsic amplitudes. In spite of this, one may expect that, on average, modes of higher $\ell$ would tend to have lower apparent amplitudes. PG 1336-018 turns out to be interesting at this level too, since the relatively high number of available periods (25) provide, for the first time in the field, a relatively meaningful basis for such statistical arguments. We note, in particular, that the measured amplitudes for the fitted periods given in Table 3 show the expected qualitative trend: according to the inferred mode identification, the observed average amplitudes, $\bar{A}_{\ell}$, are: $\bar{A}_{0}=0.200 \%$ (for $\ell=0$ modes), $\bar{A}_{1}=0.196 \%$ (for $\ell=1$ modes), $\bar{A}_{2}=0.166 \%$ (for $\ell=2$ modes), and $\bar{A}_{4}=0.096 \%$ (for $\ell=4$ modes). The significant drop of average amplitude for the periods identified as $\ell=4$ modes is particularly striking and corresponds well, at least qualitatively, to the theoretical expectation that there is, indeed, a stronger visibility penalty for modes with $\ell=4$ compared to modes having $\ell \leq 2$ (see, Randall et al. 2005a, 2007).

The fair consistency achieved by our optimal model at the level of the mode amplitude hierarchy is another argument contributing to the overall credibility of this seismic solution for PG 1336-018. As a matter of comparison, we mentioned in Sect. 3.4 (see also Fig. 3) that a few regions of the model parameter space could provide acceptable models in terms strictly 
Table 3. Period fit and derived mode identification for the optimal model of PG 1336-018. Only the relevant theoretical modes are shown in this table, due to space limitations.

\begin{tabular}{|c|c|c|c|c|c|c|c|c|c|c|c|}
\hline$l$ & $k$ & $m$ & $\begin{array}{c}v_{\mathrm{obs}} \\
(\mu \mathrm{Hz})\end{array}$ & $\begin{array}{c}v_{\mathrm{th}} \\
(\mu \mathrm{Hz})\end{array}$ & $\begin{array}{l}P_{\text {obs }} \\
(\mathrm{s})\end{array}$ & $\begin{array}{l}P_{\text {th }} \\
(\mathrm{s})\end{array}$ & $\begin{array}{c}\Delta X / X \\
(\%)\end{array}$ & $\begin{array}{l}\Delta P \\
(\mathrm{~s})\end{array}$ & $\begin{array}{c}\Delta v \\
(\mu \mathrm{Hz})\end{array}$ & $\begin{array}{c}\text { Ampl. } \\
(\%)\end{array}$ & Comments \\
\hline 0 & 1 & 0 & & 5827.935 & $\ldots$ & 171.5874 & $\ldots$ & $\ldots$ & $\ldots$ & $\ldots$ & \\
\hline 0 & 0 & 0 & 5435.373 & 5439.147 & 183.9800 & 183.8524 & +0.0694 & +0.1276 & -3.773 & 0.20 & $f_{5}$ \\
\hline 1 & 2 & -1 & 6163.328 & 6143.287 & 162.2500 & 162.7793 & -0.3262 & -0.5293 & +20.041 & 0.05 & $f_{28}$ \\
\hline 1 & 2 & 0 & $\ldots$ & 6032.043 & $\ldots$ & 165.7813 & $\ldots$ & $\ldots$ & $\ldots$ & $\ldots$ & \\
\hline 1 & 2 & +1 & 5916.110 & 5920.798 & 169.0300 & 168.8961 & +0.0792 & +0.1339 & -4.689 & 0.10 & $f_{11}$ \\
\hline 1 & 1 & -1 & 5585.656 & 5586.476 & 179.0300 & 179.0037 & +0.0147 & +0.0263 & -0.820 & 0.40 & $f_{2}$ \\
\hline 1 & 1 & 0 & 5470.759 & 5474.019 & 182.7900 & 182.6811 & +0.0596 & +0.1089 & -3.260 & 0.06 & $f_{22}$ \\
\hline 1 & 1 & +1 & 5369.416 & 5361.562 & 186.2400 & 186.5128 & -0.1465 & -0.2728 & +7.854 & 0.37 & $f_{3}$ \\
\hline 2 & 3 & -2 & $\ldots$ & 7401.844 & $\ldots$ & 135.1015 & $\ldots$ & $\ldots$ & $\ldots$ & $\ldots$ & \\
\hline 2 & 3 & -1 & $\ldots$ & 7290.492 & $\ldots$ & 137.1649 & $\ldots$ & $\ldots$ & $\ldots$ & $\ldots$ & \\
\hline 2 & 3 & 0 & $\ldots$ & 7179.141 & $\ldots$ & 139.2924 & $\ldots$ & $\ldots$ & $\ldots$ & $\ldots$ & \\
\hline 2 & 3 & +1 & 7071.136 & 7067.789 & 141.4200 & 141.4870 & -0.0474 & -0.0670 & +3.347 & 0.13 & $f_{9}$ \\
\hline 2 & 3 & +2 & $\ldots$ & 6956.437 & $\ldots$ & 143.7518 & $\ldots$ & $\ldots$ & $\ldots$ & $\ldots$ & \\
\hline 2 & 2 & -2 & $\ldots$ & 6677.528 & $\ldots$ & 149.7560 & $\ldots$ & $\ldots$ & $\ldots$ & $\ldots$ & \\
\hline 2 & 2 & -1 & $\ldots$ & 6570.556 & $\ldots$ & 152.1941 & $\ldots$ & $\ldots$ & $\ldots$ & $\ldots$ & \\
\hline 2 & 2 & 0 & $\ldots$ & 6463.583 & $\ldots$ & 154.7129 & $\ldots$ & $\ldots$ & $\ldots$ & $\ldots$ & \\
\hline 2 & 2 & +1 & $\ldots$ & 6356.611 & $\ldots$ & 157.3165 & $\ldots$ & $\ldots$ & $\ldots$ & $\ldots$ & \\
\hline 2 & 2 & +2 & $\ldots$ & 6249.639 & $\ldots$ & 160.0092 & $\ldots$ & $\ldots$ & $\ldots$ & $\ldots$ & \\
\hline 2 & 1 & -2 & 5757.384 & 5742.918 & 173.6900 & 174.1275 & -0.2519 & -0.4375 & +14.466 & 0.47 & $f_{1}$ \\
\hline 2 & 1 & -1 & 5621.135 & 5630.979 & 177.9000 & 177.5890 & +0.1748 & +0.3110 & -9.844 & 0.07 & $f_{20}$ \\
\hline 2 & 1 & 0 & 5516.633 & 5519.041 & 181.2700 & 181.1909 & +0.0436 & +0.0791 & -2.409 & 0.10 & $f_{12}$ \\
\hline 2 & 1 & +1 & 5401.026 & 5407.103 & 185.1500 & 184.9419 & +0.1124 & +0.2081 & -6.077 & 0.06 & $f_{25}$ \\
\hline 2 & 1 & +2 & $\ldots$ & 5295.165 & $\ldots$ & 188.8515 & $\ldots$ & $\ldots$ & $\ldots$ & $\ldots$ & \\
\hline 4 & 5 & 0 & $\ldots$ & 10387.173 & $\ldots$ & 96.2726 & $\ldots$ & $\ldots$ & $\ldots$ & $\ldots$ & \\
\hline 4 & 5 & +1 & 10314.595 & 10276.775 & 96.9500 & 97.3068 & -0.3680 & -0.3568 & +37.820 & 0.05 & $f_{26}$ \\
\hline 4 & 5 & +2 & $\ldots$ & 10166.377 & $\ldots$ & 98.3635 & $\ldots$ & $\ldots$ & $\ldots$ & $\ldots$ & \\
\hline 4 & 3 & -2 & $\ldots$ & 8178.214 & $\ldots$ & 122.2761 & $\ldots$ & $\ldots$ & $\ldots$ & $\ldots$ & \\
\hline 4 & 3 & -1 & $\ldots$ & 8071.613 & $\ldots$ & 123.8910 & $\ldots$ & $\ldots$ & $\ldots$ & $\ldots$ & \\
\hline 4 & 3 & 0 & 7948.494 & 7965.012 & 125.8100 & 125.5491 & +0.2074 & +0.2609 & -16.518 & 0.06 & $f_{24}$ \\
\hline 4 & 3 & +1 & 7880.842 & 7858.411 & 126.8900 & 127.2522 & -0.2854 & -0.3622 & +22.431 & 0.07 & $f_{19}$ \\
\hline 4 & 3 & +2 & $\ldots$ & 7751.810 & $\ldots$ & 129.0021 & $\ldots$ & $\ldots$ & $\ldots$ & $\ldots$ & \\
\hline 4 & 2 & -4 & 7412.898 & 7414.010 & 134.9000 & 134.8798 & +0.0150 & +0.0202 & -1.111 & 0.06 & $f_{23}$ \\
\hline 4 & 2 & -3 & $\ldots$ & 7303.060 & $\ldots$ & 136.9289 & $\ldots$ & $\ldots$ & $\ldots$ & $\ldots$ & \\
\hline 4 & 2 & -2 & $\ldots$ & 7192.111 & $\ldots$ & 139.0412 & $\ldots$ & $\ldots$ & $\ldots$ & $\ldots$ & \\
\hline 4 & 2 & -1 & 7108.836 & 7081.161 & 140.6700 & 141.2198 & -0.3908 & -0.5498 & +27.675 & 0.09 & $f_{14}$ \\
\hline 4 & 2 & 0 & $\ldots$ & 6970.211 & $\ldots$ & 143.4677 & $\ldots$ & $\ldots$ & $\ldots$ & $\ldots$ & \\
\hline 4 & 2 & +1 & $\ldots$ & 6859.262 & $\ldots$ & 145.7883 & $\ldots$ & $\ldots$ & $\ldots$ & $\ldots$ & \\
\hline 4 & 2 & +2 & $\ldots$ & 6748.312 & $\ldots$ & 148.1852 & $\ldots$ & $\ldots$ & $\ldots$ & $\ldots$ & \\
\hline 4 & 1 & -4 & $\ldots$ & 6104.366 & $\ldots$ & 163.8172 & $\ldots$ & $\ldots$ & $\ldots$ & $\ldots$ & \\
\hline 4 & 1 & -3 & $\ldots$ & 5997.193 & $\ldots$ & 166.7447 & $\ldots$ & $\ldots$ & $\ldots$ & $\ldots$ & \\
\hline 4 & 1 & -2 & 5891.363 & 5890.020 & 169.7400 & 169.7787 & -0.0228 & -0.0387 & +1.343 & 0.09 & $f_{15}$ \\
\hline 4 & 1 & -1 & $\ldots$ & 5782.847 & $\ldots$ & 172.9252 & $\ldots$ & $\ldots$ & $\ldots$ & $\ldots$ & [close to $f_{8}$ ] \\
\hline 4 & 1 & 0 & $\ldots$ & 5675.674 & $\ldots$ & 176.1905 & $\ldots$ & $\ldots$ & $\ldots$ & $\ldots$ & \\
\hline 4 & 1 & +1 & $\ldots$ & 5568.500 & $\ldots$ & 179.5816 & $\ldots$ & $\ldots$ & $\ldots$ & $\ldots$ & [close to $f_{13}$ ] \\
\hline 4 & 1 & +2 & 5444.251 & 5461.327 & 183.6800 & 183.1057 & +0.3127 & +0.5743 & -17.076 & 0.17 & $f_{7}$ \\
\hline 4 & 1 & +3 & 5356.473 & 5354.154 & 186.6900 & 186.7709 & -0.0433 & -0.0809 & +2.319 & 0.08 & $f_{16}$ \\
\hline 4 & 1 & +4 & $\ldots$ & 5246.981 & $\ldots$ & 190.5858 & $\ldots$ & $\ldots$ & $\ldots$ & $\ldots$ & \\
\hline 4 & 0 & -4 & $\ldots$ & 5706.791 & $\ldots$ & 175.2298 & $\ldots$ & $\ldots$ & $\ldots$ & $\ldots$ & \\
\hline 4 & 0 & -3 & 5598.477 & 5606.049 & 178.6200 & 178.3787 & +0.1351 & +0.2413 & -7.572 & 0.17 & $f_{6}$ \\
\hline 4 & 0 & -2 & 5505.698 & 5505.307 & 181.6300 & 181.6429 & -0.0071 & -0.0129 & +0.391 & 0.08 & $f_{17}$ \\
\hline 4 & 0 & -1 & 5392.289 & 5404.565 & 185.4500 & 185.0288 & +0.2271 & +0.4212 & -12.276 & 0.25 & $f_{4}$ \\
\hline 4 & 0 & 0 & $\ldots$ & 5303.823 & $\ldots$ & 188.5432 & $\ldots$ & $\ldots$ & $\ldots$ & $\ldots$ & \\
\hline 4 & 0 & +1 & 5218.934 & 5203.081 & 191.6100 & 192.1938 & -0.3047 & -0.5838 & +15.853 & 0.06 & $f_{21}$ \\
\hline 4 & 0 & +2 & 5111.168 & 5102.340 & 195.6500 & 195.9885 & -0.1730 & -0.3385 & +8.828 & 0.05 & $f_{27}$ \\
\hline 4 & 0 & +3 & $\ldots$ & 5001.598 & $\ldots$ & 199.9361 & $\ldots$ & $\ldots$ & $\ldots$ & $\ldots$ & \\
\hline 4 & 0 & +4 & 4885.198 & 4900.856 & 204.7000 & 204.0460 & +0.3195 & +0.6540 & -15.658 & 0.07 & $f_{18}$ \\
\hline
\end{tabular}


of the quality of fit to the observed periods (i.e., within 3- $\sigma$ in $S^{2}$ value relative to the best-fit model), while being also consistent with the spectroscopic constraints. However, for all these regions, the resulting mode identifications are much less convincing regarding the hierarchy of apparent mode amplitudes. The region near $M \sim 0.64 M_{\odot}$ provides a seismic model candidate with an inconsistent distribution for the average amplitude values: $\bar{A}_{\ell}=\{0.065,0.073,0.209,0.115 \%\}$ (for $\ell=\{0,1$, $2,4\}$, respectively). The $M \sim 0.36 M_{\odot}$ solution results in values $\bar{A}_{\ell}=\{0.170,0.167,0.190,0.082 \%\}$ and is less convincing (since $\bar{A}_{2}>\bar{A}_{0}, \bar{A}_{1}$ ), despite the fact that the periods identified as $\ell=4$ modes have clearly lower amplitudes. The family of models near $M \sim 0.43 M_{\odot}$ provides a period fit with $\bar{A}_{\ell}=\{0.170$, $0.220,0.120,0.114 \%$ which, again, is not very satisfactory in terms of amplitude hierarchy. Finally, the $M \sim 0.47 M_{\odot}$ solution is in fact very close to our optimal solution parameter-wise, but provides a significantly different distribution of the $\left(P_{\mathrm{obs}}, P_{\mathrm{th}}\right)$ pairs in the resulting period fit. The average amplitude distribution in that case is $\bar{A}_{\ell}=\{0.215,0.082,0.216,0.111 \%\}$, i.e., less credible. Hence, based on these specific arguments, we find that our optimal model solution is also the one that provides the most consistent mode identification in terms of preserving statistically the expected hierarchy of apparent mode amplitudes. This consideration adds up to the other elements mentioned previously that conducted us to favor this model as the best asteroseismic solution for PG 1336-018.

Finally, it is interesting to look back at the three uncertain periods from the original set of Kilkenny et al. (2003) that were not considered for the search of a best-fit asteroseismic model (see Sect. 3.1 and Table 1), and check if these have counterparts in our optimal seismic model of PG 1336-018. These periods are $184.04 \mathrm{~s}\left(f_{10}\right), 178.96 \mathrm{~s}\left(f_{13}\right)$, and $173.59 \mathrm{~s}\left(f_{8}\right)$. We find that $f_{10}$ has no acceptable counterpart in the proposed model, but both $f_{13}$ and $f_{8}$ can be associated with unassigned theoretical periods. Indeed, $f_{13}$ can be associated with the mode $\ell=4, k=1, m=+1$ that has a period of $\sim 179.58 \mathrm{~s}$ (see Table 3 ). This match has a relative dispersion of $\Delta P / P=-0.35 \%(\Delta P=-0.62 \mathrm{~s}$, on an absolute scale). For its part, $f_{8}$ can be associated with the mode $\ell=4, k=1, m=-1$ that has a period of $\sim 172.92 \mathrm{~s}$. This match has a relative dispersion of $\Delta P / P=+0.39 \%(\Delta P=+0.67 \mathrm{~s}$, on an absolute scale). Hence, these two a posteriori period matches show dispersions comparable to the other fitted periods and do not affect significantly the overall quality of the fit if included. This suggests that $f_{13}$ and $f_{8}$ may well be real oscillation modes of the star, a proposition that would require additional observations, however, to be confirmed.

\subsection{On the orientation of the pulsation axis}

An interesting and unexpected byproduct of the present asteroseismic analysis is related to the derived mode identification for the 25 periods observed in PG 1336-018. Table 3 shows that 5 observed periods have been identified as components of two $\ell=1$ triplets of radial order $k=1$ and 2 . In these triplets, it turns out that the periods with the highest observed amplitudes are associated with the $m= \pm 1$ components. This is particularly striking for the $\ell=1, k=1$ triplet where the $m=+1,-1$ components have amplitudes of 0.37 and $0.40 \%$ respectively, while the central $m=0$ component has an amplitude of $0.06 \%$, only. In the same vain, the $\ell=1, k=2$ triplet has only the two $m=-1$ and +1 components associated with observed periods of rather low amplitudes ( 0.05 and $0.10 \%$ respectively), while the central $m=0$ mode is not seen in that case (its amplitude could very well be below the detection limit of the current data).

It is well known from stellar pulsation theory that the visibility of modes of azimuthal order $m$ for unresolved stellar disks is modulated by the orientation of the pulsation axis relative to the observer. For instance, an $\ell=1, m=0$ mode, whose geometry imposes an equatorial node between two hemispheres in opposition of phase during a pulsation cycle, will be severely attenuated by disk-averaging effects if the pulsations are seen nearly equator on. Conversely, such a mode would suffer only weak attenuation if the pulsations are seen pole on by the observer. The situation is reversed for the $\ell=1$ modes having $m= \pm 1$ azimuthal orders, whose geometry imposes, this time, a longitudinal node between regions of opposite phase.

In the present situation, the amplitude configuration observed for the dipole $(\ell=1)$ modes is favored if the pulsations are seen nearly equator on (i.e., at a high inclination angle). In that case, strong attenuation of the central $m=0$ components are likely to lower significantly their observable amplitudes relative to the $m= \pm 1$ components, as it is apparently the case according to Table 3 . The case for a high inclination angle is further strengthened by invoking the relative amplitudes of the four components of the multiplet corresponding to the modes with $\ell=2$ and $k=1$. Indeed, a quadrupole observed nearly equator on and split by rotation will tend to show $|m|=2$ components with higher amplitudes than the central $m=0$ component, while the $|m|=1$ components tend to be invisible (see, e.g., Fig. 1 of Brassard et al. 1995). This is exactly what is suggested in Table 3, although the $m=+2$ component is not seen for some reason in that quintuplet. The interesting point about this discussion of the inclination angle is that we already know that the eclipsing binary system PG $1336-018$ is seen nearly equator on, with an inclination angle of $\sim 81^{\circ}$ well constrained from the binary light curve analysis of Vučković et al. (2007). Hence, this very strongly suggests that the pulsation axis is aligned with the rotation axis of the sdB star in PG 1336-018, the latter being most likely perpendicular to the orbital plane as must be the case in such a close binary system.

This result is enlightening in view of recent claims to the effect that the pulsation axis could be tipped toward the companion star in close binary systems such as PG 1336-018 (Reed et al. 2006, and references therein). It was indeed argued, although there was no credible theoretical foundations for this proposition to be made, that tidal effects could tip the pulsation axis of the sdB star toward the companion and into the orbital plane. Such a tipped axis would then precess with the rotation of the star and produce all sorts of complications that would render an asteroseismic interpretation of the light curve nearly impossible. Our results show, on the contrary, that PG 1336-018 is amenable to detailed studies. In particular, they demonstrate that the pulsation axis of that star is not tipped as argued by some authors.

\section{Expanding the limits of asteroseismology}

\subsection{Relaxing the spin-orbit synchronism hypothesis}

One underlying hypothesis imposed in the previous asteroseismic analysis of PG $1336-018$ is that the rotation of the sdB star is fully synchronized with its orbital motion, i.e., from the stellar surface down to the central region. The star was therefore assumed to rotate as a solid body with a period, $P_{\text {rot }}$, equal to $\sim 8728$ s, i.e., identical to the measured orbital period of the system. The properties of the asteroseismic solution presented previously and inferred within the framework of this assumption 
strongly suggest at this stage that the star is indeed fully synchronized. As an additional independent check and in the spirit of relaxing this hypothesis, we also operated a search in the entire model parameter space still assuming rigid rotation, but leaving this time $P_{\text {rot }}$ as a free parameter. Not surprisingly, the same families of model solutions which were found in Sect. 3 emerged from this exploration, all with values for the period $P_{\text {rot }}$ very close (i.e., within $1-\sigma$ ) to the orbital period of the system. This further strengthen the fact that PG $1336-018$ is very likely in synchronous rigid rotation. However, the idea of complete spinorbit synchronism deserves more attention in our view, and we further investigate it in what follows.

The theoretical framework describing tidal frictions in close binaries has been developed essentially by Zahn (1977) and Tassoul \& Tassoul (1992a,b). These studies show that synchronization times depend notably on the orbital period, but can differ by orders of magnitude depending on the physical mechanism invoked to insure synchronism. This is particularly true for hot stars with radiative envelopes (such as sdB stars), where tidal forces are believed to be less efficient for synchronization. While the mechanism of Zahn is not dissipative enough to explain the observed levels of synchronization, the validity of the mechanism proposed by Tassoul \& Tassoul is still under debate, given its free parameter dependence (see, Claret et al. 1995; Claret \& Cunha 1997). Independently of these theoretical uncertainties, observational evidence already exists in favor of the idea that very close systems such as the sdB+WD binaries KPD 0422+5421 (Orosz \& Wade 1999) and KPD $1930+2752$ (Billères et al. 2000), with orbital periods of $\sim 2.16 \mathrm{~h}$ and $\sim 2.28 \mathrm{~h}$, respectively, have achieved spin-orbit synchronism, at least superficially, over the typical lifetime of a sdB star $\left(\sim 10^{8} \mathrm{yrs}\right)$, since both systems show ellipsoidal luminosity variations with a period of exactly half the orbital period, as expected for tidally locked binaries. The hypothesis of spin-orbit synchronism was indeed used recently by Geier et al. (2007) to constrain the binary parameters of KPD $1930+2752$ and, in a more recent work, Geier et al. (2008) also presented a study of a third sdB+WD system, PG $0101+039$, for which they were able to show that the tiny ellipsoidal variation has a period that is, again, equal to half the orbital period of $13.68 \mathrm{~h}$. These results suggest that tidal synchronization, at least for the outermost stellar layers, is achieved in sdB+WD systems with orbital periods of less than $\sim 14 \mathrm{~h}$. Remarkably, asteroseismology has recently taken hold of these questions too, by providing very strong evidence that another sdB+WD system, Feige 48 having an orbital period of $\sim 9.02 \mathrm{~h}$, is tidally locked but also rotates most likely as a solid body (Van Grootel et al. 2008a). This pioneering work is the first explicit demonstration of spin-orbit synchronism in a binary star by asteroseismic means.

On the basis of this evidence, there is little doubt that the outermost layers (the surface at the very least) of the sdB primary in PG 1336-018 should be tidally locked in this close system. However, our knowledge of the dynamical status of the inner layers of the star is a lot more uncertain. In this context, it appears highly legitimate to challenge, if possible, the hypothesis that a star can really rotate as a solid body. There is, indeed, a more general and highly interesting question linked to this situation: how deep can tidal spin-orbit synchronization migrate inside stars within their lifetime? It turns out that PG 1336-018 is also a particularly interesting laboratory for addressing that specific issue, as we shall see below.

In order to explore this particular question, we relaxed the strict spin-orbit synchronism hypothesis adopted in the preceding analysis. Specifically, we gave up the simple solid-body rotation law that was used in the computation of the rotationally split pulsation frequencies and replaced it with a spherically symmetric differential rotation law (we do not consider axisymmetric differential rotation here). In this more general context, Eqs. (1) and (3) are no longer valid and must be replaced by the general relations

$\Delta v_{k \ell}=\int_{0}^{R} V_{\text {rot }}(r) K_{k \ell}(r) \mathrm{d} r$

where $V_{\text {rot }}(r) \equiv \Omega_{\text {rot }}(r) / 2 \pi=1 / P_{\text {rot }}(r)$ is the rotation rate of the star (in $\mathrm{Hz}$ ), now a function of the radius $r$, and $K_{k \ell}(r)$ is the first-order rotation kernel computed from the mode eigenfunctions $\xi_{r}(r)$ and $\xi_{h}(r)$ :

$K_{k \ell}(r)=\frac{\xi_{r}^{2}-[\ell(\ell+1)-1] \xi_{h}^{2}-2 \xi_{r} \xi_{h}}{\int_{0}^{R}\left\{\xi_{r}^{2}+\ell(\ell+1) \xi_{h}^{2}\right\} \rho r^{2} d r} \rho r^{2}$

Equation (2) then applies to derive the first-order corrections to the eigenfrequencies. In this exploration, we considered a simple differential rotation law characterized by two regions the "core" and the "envelope" - rotating each as independent solid structures. The position of the transition (or sheer) layer between the "core" and the "envelope" is specified by the parameter $R_{\mathrm{S}} / R_{*}$ (i.e., the radius expressed as a fraction of the total radius of the star, $R_{*}$ ). Two other parameters are needed to specify fully this rotation law. These are the rotation period for the envelope, $P_{\text {env }}$ (in seconds), and the rotation period for the core, $P_{\text {core }}$ (also in seconds). For PG 1336-018, we further assumed that the "envelope" is tidally synchronized and therefore the parameter $P_{\text {env }}$ was fixed to the value of the orbital period, i.e., 8727.78 s. The two other parameters were varied, in the ranges 1000-19000 s (by steps of $200 \mathrm{~s}$ ) and $0.2-1.0$ (by steps of 0.01 ) for $P_{\text {core }}$ and $R_{\mathrm{s}} / R_{*}$, respectively. Since $R_{\mathrm{S}} / R_{*}$ is a free parameter, we stress that what we call the "envelope" in this context is the region between the surface $\left(r / R_{*}=1.0\right)$ and this "sheer layer" placed at the position $R_{\mathrm{S}} / R_{*}$. The so-called "core" is therefore the region below this transition. We also note that the particular case $R_{\mathrm{s}} / R_{*}=1.0$, considered in our grid exploration, is equivalent to a solid body rotation law of period $P=P_{\text {core, }}$, where this time $P$ is a free parameter contrary to the analysis of Sect. 3 . Finally, in this exercise, we kept all other structural parameters of the star $\left(T_{\mathrm{eff}}\right.$, $\log g, \log q(H)$, and $M_{*}$ ) constant, set to the firmly established values derived formerly from our optimal seismic model solution. Indeed, we consider from that point that these parameters have been well secured owing to the fact that both the asteroseismic and the orbital solutions independently point toward these values. Figure 4 shows the resulting $S^{2}$ map in the 2-dimensional $\left(P_{\text {core }}, R_{\mathrm{S}} / R_{*}\right)$ parameter space explored.

At high values of $R_{\mathrm{S}} / R_{*}(\gtrsim 0.55)$, we find a very well defined valley of low $S^{2}$ values corresponding to the models that best fit the 25 periods of PG 1336-018. Interestingly, we find that this valley is centered around a rotation period $P_{\text {core }}$ corresponding to the orbital period of the system (the dot-dashed vertical line in Fig. 4). This means that, even with the additional freedom offered by the new rotation law considered, best-fit models spontaneously converge toward tidally synchronized solid body rotation, at least down to $\sim 0.55 R_{*}$ inside the star. Indeed, the fact that the bottom of the valley, indicated by the white solid-curve in Fig. 4, matches almost exactly the dot-dashed vertical line in that radius range is particularly suggestive. In addition, we note that this solution is unique, as the quality of the fit rapidly degrades for models that differ significantly from that rotation 


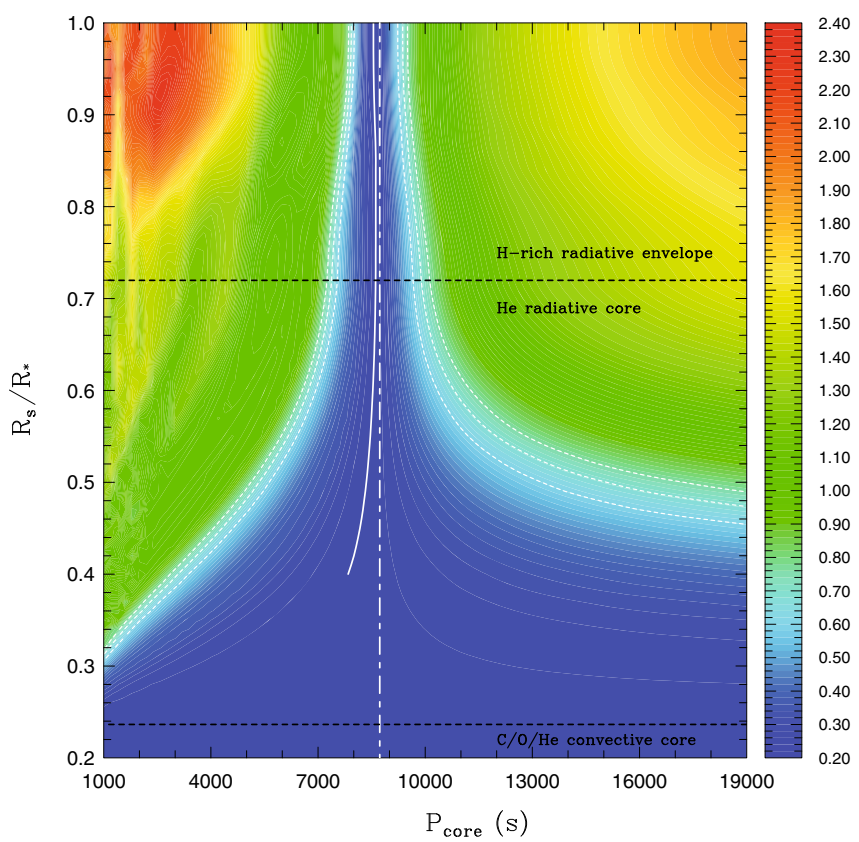

Fig. 4. Seismic inversion of the internal rotation profile of the sdB star in PG 1336-018. This $S^{2}$ map (on a logarithmic scale) shows the quality of fit to the observed pulsation periods as a function of the parameters $P_{\text {core }}$ and $R_{S} / R_{*}$ that define the differential rotation law explored (see text for details). The white dot-dashed vertical line indicates the rotation period of PG 1336-018 assuming a fully synchronized star and, therefore, pure solid body rotation with a period of $\sim 8728 \mathrm{~s}$. The white solid curve represents the line of minimum $S^{2}$ in this map for $R_{S} / R_{*}$ in the range 0.4-1.0. White dotted-line contours show regions where the period fits have $S^{2}$ values within, respectively, the $1-\sigma, 2-\sigma$, and $3-\sigma$ confidence level relative to the best-fit solution. Finally, the black horizontal dotted-lines show, respectively, the location of the transition between the H-rich envelope and the helium core in our optimal stellar model (at $R / R \sim 0.72$ ) and the typical location of the boundary of the inner, convective He-burning core according to standard evolutionary models (see, e.g., Dorman et al. 1993).

profile. Hence, alternative rotation periods for the sdB primary star in PG 1336-018 can clearly be excluded, at least in the explored period range. This finding is quite consistent with the fact that our optimal solution identified in Sect. 3 already matches the 25 observed periods with an accuracy comparable, from our past experience, to the best that can be achieved in sdB asteroseismology with current models. Consequently, there should be practically no room left for further improvements at that level based on such models.

For values of $R_{\mathrm{S}} / R_{*} \lesssim 0.55$, the valley enlarges significantly, as the 1-, 2-, and 3- $\sigma$ confidence levels represented by the white dotted-curves in Fig. 4 diverge to both shorter and longer periods. This means that, as we move the "sheer layer" deeper and deeper inside the star, models with $P_{\text {core }}$ values increasingly different from the orbital period can no longer be formally distinguished from the pure solid body rotation solution. This behavior is easily understood by recalling that acoustic modes in hot B subdwarfs are envelope modes, propagating mostly in the outer stellar regions (see, e.g., Charpinet et al. 2000). These modes have amplitudes that decrease significantly in the core to the extent of becoming unaffected by the innermost regions. Hence, the shape of the valley observed in Fig. 4 is simply the signature of the $p$-modes observed in PG 1336-018 losing progressively their sensitivity to the stellar innermost layers. The bottom line is that it becomes formally impossible, at this stage, to state whether PG $1336-018$ is in synchronized solid body rotation in its deepest regions or not. Nonetheless, we point out that near $R_{\mathrm{S}} / R_{*} \sim 0.6$ and deeper, the bottom of the valley shows a slight inflection toward shorter periods. Such a trend, if real, would indicate that the star is no longer synchronized and rotating as a solid body in these regions and below. But, again, this cannot be formally established, as the differences of $S^{2}$ values are not significant.

To summarize the results of this experiment, we found a very clear asteroseismic evidence that the sdB primary in $\mathrm{PG}$ 1336-018 has a solid-body rotation profile with a period equal to the orbital period of the system from its surface down to $\sim 0.5-0.6 R_{*}$, at least. This means that spin-orbit synchronization mechanisms have been effective for tidally locking this star over approximately half its radius, at least. This range includes the whole H-rich outer envelope of the star - its inner boundary being located near $\sim 0.72 R_{*}$ according to our optimal seismic model of PG 1336-018 - and the outermost layers of the $\mathrm{He}$ radiative core (see Fig. 4). The dynamical status of the deeper layers is more uncertain, however, due to the fact that $p$-modes probe mainly the outer layers of sdB stars and loose their sensitivity to the innermost regions. Synchronized solid body rotation extending to deeper regions (and even down to the center of the star) remains a valid option but cannot be verified. Alternatively, a very slight trend indicates that solid body rotation and tidal synchronization might break down below $\sim 0.55 R_{*}$, the rotation rate slowly increasing downward, but this also cannot be formally proved and should be taken with great caution.

Finally, we mention that, to our knowledge, this is the first time that such an explicit seismic inversion of the inner rotation profile of a star other than the Sun has been successfully carried out. Approximate inferences of internal rotation properties of some pulsating stars already exist in the litterature, most notably for a few $\beta$-Cephei stars showing rotationally-split components for both a "shallow" $p$-mode probing mostly the envelope and a "deep" $g$-mode probing the core region (Pamyatnykh et al. 2004; Dupret et al. 2004; Briquet et al. 2007). These were limited, however, by the small number of frequencies available (arguably compensated by the fact that partial independent mode identification of $\ell$ and $m$ was available in those cases). At the outset, PG 1336-018, with up to 15 modes constraining the rotation (while 10 modes determine the internal structure itself; see, again, Table 3), demonstrated a remarkable potential in that respect. This potential can possibly be exploited further when sdB stellar models become more realistic, and when our ability to fit the pulsation periods at an even higher level of accuracy still improves. We might then be able to constrain more tightly the rotation profile and extract useful information on the dynamics of even deeper regions. On a more general note, this new result is a clear demonstration that asteroseismology is indeed a very powerful tool to probe stellar structures and internal dynamics. This has very often been claimed in the past, but we offer here an explicit example of such realization.

\subsection{Higher-order effects due to rotation and tidal distortion}

For PG 1336-018 which rotates fairly rapidly and has a close companion, questions related to the importance of higher-order effects due to rotation and tidal distortions are legitimate. However, this is not a trivial problem since, unlike first-order corrections used in the present analysis, there is currently no simple formulation that would allow us to compute precisely second-order (and above) effects for moderately rotating $\mathrm{sdB}$ pulsators. In the present context, the goal is first to evaluate the 


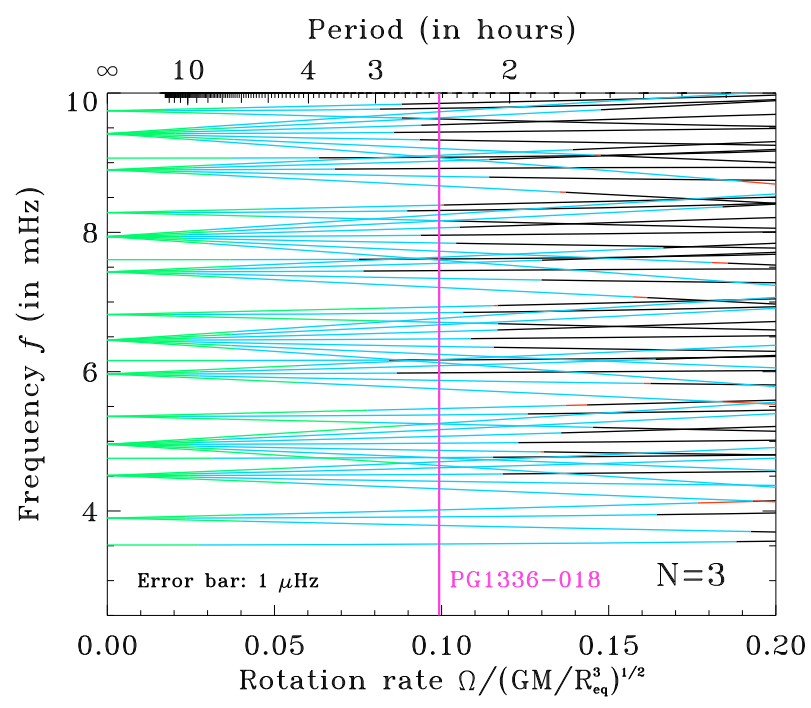

Fig. 5. Modes of degree $\ell=0-3$ in the frequency range $3-10 \mathrm{mHz}$ are shown as a function of the rotation rate of the star (in units of the breakup rotation rate $\Omega_{K} \equiv \sqrt{G M / R_{\mathrm{eq}}^{3}}$ ). Parameters relevant to PG 1336-018 were used in this calculation $\left(M=0.459 M_{\odot}\right.$ and $R=0.15 R_{\odot}$ ). The range of validity for each level of approximation, defined as the range where the difference with the exact frequency (i.e., computed in a nonperturbative way) is less than the precision of the observed frequencies (fixed to $1 \mu \mathrm{Hz}$ in this case), is indicated by the color segments: green, cyan, and red represent the first-order, secondorder, and third-order approximations, respectively. The black portions indicate regions where effects of higher-order show-up above the $1 \mu \mathrm{Hz}$ limit. PG 1336-018, assuming a rotation period of $2.4244 \mathrm{~h}$, lies near $\Omega / \Omega_{K} \sim 0.1$ in this diagram.

eventual significance of these corrections and determine if this lack would gain to be filled in the future.

To progress in this area, we exploited the tools recently developed, in part, by one of us (D. R.) to study the behavior of oscillation modes in rapidly rotating stars. This original approach, described in detail in Lignières et al. (2006) and Reese et al. (2006), relies on a nonperturbative treatment of stellar rotation that provides "exact" solutions for the frequencies of the modes. By "exact", we mean that the full set of equations describing this problem is solved and no approximate development of the solution is made, contrary to the perturbative approach. The projection of these "exact" solutions on the $\left\{\Omega^{1}, \Omega^{2}, \ldots, \Omega^{n}\right\}$-basis leads, after truncation at the $n$th power of $\Omega$, to the solution approximated at the $n$ th-order whose precision can then be estimated. However, the current trade-off in these exact calculations is that the method has not yet been applied to realistic stellar structures and relies instead on approximate representations of stars based on polytropes. Nonetheless, although precise numerical evaluations of the rotation effects very likely remain impaired by this limitation, interesting insight on the importance of such effects in the context of PG 1336-018 can still be gained, at least at the level of providing orders of magnitudes for the various effects.

Figure 5 shows the expected behavior of the relevant $p$-mode frequencies in a star like PG $1336-018$ (i.e., in the $3-10 \mathrm{mHz}$ range) using this nonperturbative method to evaluate the effects of rotation. It also indicates the range of validity of various orders of approximation related to the standard perturbative approach (see the figure caption for details). In this calculation, the sdB star structure was approximated by a polytrope of index $N=3$ roughly representative of the outermost layers of this type of stars where acoustic waves predominantly exist. A mass of $0.459 M_{\odot}$ and an equatorial radius of $0.151 R_{\odot}$ corresponding to the values derived in Sect. 3 from our asteroseismic analysis were assumed in that context. Figure 5 immediately indicates that in PG 1336-018, a rotation period of $2.4244 \mathrm{~h}$ produces higher-order and most notably second-order corrections to the frequencies that are larger than the precision of the observations (approximately $1 \mu \mathrm{Hz}$ ). Hence, these are expected to be significant in this star. Interestingly, the calculation indicates that the first-order perturbation is valid, strictly speaking, down to periods of $\sim 10 \mathrm{~h}$ (the green segments shown in Fig. 5), while secondorder corrections (blue segments) remain accurate at the level of the precision of the observations down to periods of 2.0-2.7 h, depending on the modes. PG 1336-018, with typical frequencies between 5 and $8 \mathrm{mHz}$, lies well within the limits of validity of the second-order corrections but relatively far above the first-order limit, thus suggesting that second-order corrections may be large enough to produce significant distortions relative to calculations limited to first-order.

In order to assess that latter point, we provide in Fig. 6 a close-up view showing the behavior of a representative nonradial mode (with $\ell=2, k=2$ ) as a function of the rotation rate of the star. For this mode, a comparison between the exact solution and the first- and second-order truncated calculations is shown in upper-left panel of Fig. 6. The separated contributions from the various levels of perturbative corrections, respectively $\delta f_{\text {pert }}^{1}, \delta f_{\text {pert }}^{2}, \delta f_{\text {pert }}^{3}$, and $\sum \delta f_{\text {pert }}^{>3}$ (given in $\mu \mathrm{Hz}$ ) for the first-, second-, third-, and the sum of all remaining perturbations, are also illustrated in the other panels of this figure. As expected, the first-order correction $\left(\delta f_{\text {pert }}^{1}\right)$ largely dominates the rotational splitting, producing an equidistant (in frequency) quintuplet with the prograde $(m<0)$ modes having their frequencies increased and the retrograde $(m>0)$ modes having their frequency decreased compared to the central $m=0$ component. As discussed already, this correction leads, for PG 1336-018 rotating with $P_{\text {rot }}=2.4244 \mathrm{~h}\left(\Omega / \Omega_{K} \sim 0.1\right)$, to a splitting between $|\Delta m|=1$ modes of $\sim 114 \mu \mathrm{Hz}$. The frequency of the axisymmetric $(m=0)$ mode is unaffected at this level of approximation. The secondorder corrections $\left(\delta f_{\text {pert }}^{2}\right)$ are all very small (i.e., irrelevant) for rotation periods beyond $\sim 10 \mathrm{~h}$, but start to significantly affect the frequencies at higher rotation rates. Figure 6 shows that these corrections, in a star rotating at the rate of PG 1336-018, could reach a magnitude of $10-15 \mu \mathrm{Hz}$ and affect unevenly the components of a multiplet. For the sample mode represented, the $m= \pm 2$ sectoral modes experience a decrease in their frequency relative to their first-order truncated frequency, while the $m= \pm 1$ modes have their frequency increased, as well as the axisymmetric $(m=0)$ mode which is now affected by the rotation of the star. Higher-order corrections remain marginal in the context of PG 1336-018. The third-order perturbations $\left(\delta f_{\text {pert }}^{3}\right)$ are well below the frequency resolution of the observations, and corrections of higher-orders all summed-up $\left(\sum \delta f_{\text {pert }}^{>3}\right)$ are not significant, except maybe for the $m=0$ component for a modest contribution of $\sim 2 \mu \mathrm{Hz}$. Finally, the lower right panel of Fig. 6 illustrates the impact on mode frequencies induced by the tidal deformation of the sdB star generated by the dwarf-M companion. This effect was estimated using the second-order perturbative formalism and coefficients of Saio (1981) and, as expected, is similar (even slightly inferior), with corrections of $\lesssim 5 \mu \mathrm{Hz}$, to the second-order corrections caused by rotation.

If Fig. 6 only illustrates the particular case of one rotationally split non radial mode - other modes will have their own specific behavior -, we stress nonetheless that the inferred 

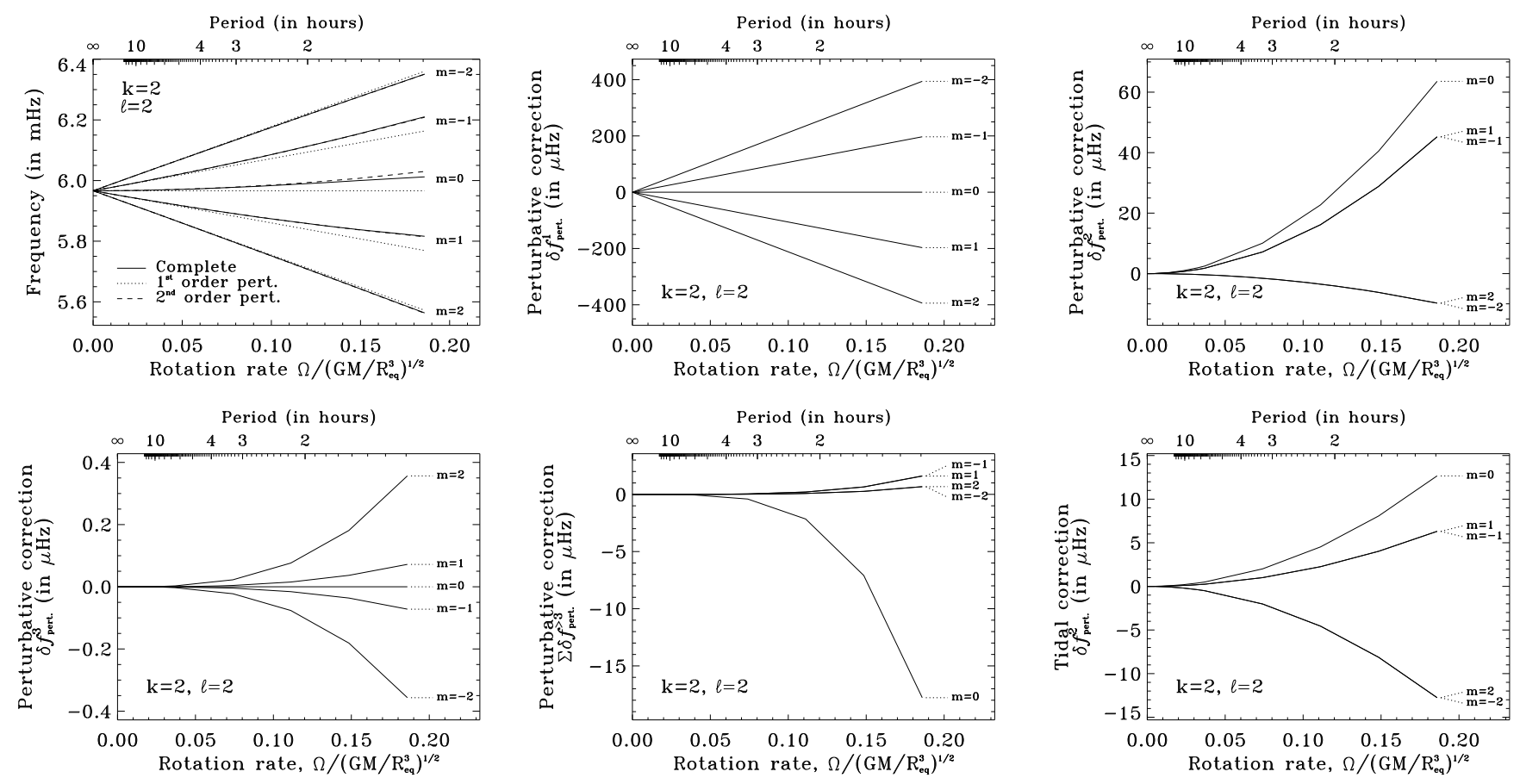

Fig. 6. In top left panel, frequencies (in $\mathrm{mHz}$ ) of a representative quintuplet (of degree $\ell=2$ and radial order $n=2$ ) vs. the rotation rate (or period) of the star are shown. The frequencies of the $m$-components are given for the exact computation (i.e., using the nonperturbative approach; solid curves), as well as in the first-order (dotted curves) and second-order (dashed curves) perturbative approximations. The individual contributions of the perturbative orders (in $\mu \mathrm{Hz}$ ) as functions of the rotation rate (or period) of the star are detailed in other panels. Top middle and right panels shows the first-order $\left(\delta f_{\text {pert }}^{1}\right)$ and second-order $\left(\delta f_{\text {pert }}^{2}\right)$ contributions to the rotational splitting, while bottom left and middle panels illustrate the contributions of the third-order $\left(\delta f_{\text {pert }}^{3}\right)$, and the sum of all remaining $\left(\sum \delta f_{\text {pert }}^{>3}\right)$ perturbations to the frequency of the quintuplet components due to rotation. Bottom right panel shows the second-order tidal correction as a function of the rotation rate (assumed synchronized with the orbital period) induced by a dwarf-M companion of $0.122 M_{\odot}$ similar to the companion of PG 1336-018.

orders-of-magnitude for the higher-order corrections provided by this example are representative (see again Fig. 5) and constitute a valuable estimate of the importance of such effects induced by the moderate rotation rate and tidal distorsion of the sdB star in PG 1336-018. In this respect, we find that these effects are, strictly speaking, significant mostly up to second-order with a typical impact of $\sim 10-15 \mu \mathrm{Hz}$ or less on the mode frequencies. Compared to the accuracy of our best fit model $(\sim 10.5 \mu \mathrm{Hz}$ on average) limited to the first-order rotation approximation, we do not expect that higher order effects will have a strong impact on the derived structural and dynamical properties of this star at the present level of accuracy. However, studies aimed at improving the seismic fit of PG 1336-018 in the future will need to accurately incorporate at least the second-order corrections.

\section{Summary and conclusion}

We presented the first detailed asteroseismic analysis performed on the primary component of the close $\mathrm{sdB}+\mathrm{dM}$ eclipsing binary system PG 1336-018 (NY Virginis). This star, a hot B subdwarf member of the EC 14026 class of nonradial pulsators, oscillates in numerous modes with periods in the 96-205 s range. As such, it has stood out as a target of choice for asteroseismic studies. The close eclipsing binary nature of PG 1336-018 also offers the opportunity to measure independently several important properties of the stars forming the system. This was exploited recently by Vučković et al. (2007) who proposed three preferred families of models that can best match the binary light curve properties. The three solutions, that the authors could not formally separate, are characterized by different masses for the sdB primary star: $M \simeq 0.389,0.466$, and $0.530 M_{\odot}($ see Table 2$)$.

We based our seismic analysis on 25 secured pulsation periods identified from a dedicated Whole Earth Telescope photometric campaign piloted by Kilkenny et al. (2003). To date, this campaign has provided the most accurate seismic data available for that star. An important component of our analysis has also been to acquire and analyze new high-quality spectra of PG 1336-018 in order to derive precise constraints for the atmospheric parameters of the pulsating $\mathrm{sdB}$ star. The seismic analysis was performed using the forward modeling approach that we have implemented for pulsating subdwarf B star asteroseismology and used quite successfully in past studies (see, e.g., Charpinet et al. 2006, and references therein). This was done using efficient optimization tools and methods developed for that purpose these past few years. This approach guaranties exhaustive searches in the explored model parameter space and objective identification of potential best-fit solutions with some information about their uniqueness. For PG 1336-018, it was necessary to incorporate the effects of rotational splitting directly in the period matching procedure, as the star is very likely tidally locked with the orbital motion in this close system, leading to a fairly rapid rotation rate. This is a recent addition to our fitting procedure that was first tested by Van Grootel et al. (2008a) in a reanalysis of the rapid pulsator Feige 48, and which now allows us to explore the dynamical properties of pulsating sdB stars. In the present study, this was done, in a first step, by considering all rotationally-split multiplet components assuming first-order perturbations induced by a solid body rotation law with a period equal to the orbital period $(P=8727.78 \mathrm{~s})$. In view of the 
properties of our optimal solution obtained within this hypothesis, this approach proved to be very conclusive, pointing effectively to the fact that the star is indeed most likely a synchronized rigid rotator. Nonetheless, in a second step, this hypothesis of solid body rotation was relaxed, leading us to propose the first explicit seismic inversion of the internal rotation profile of a star other than the Sun. Finally, we explored, for the very first time in the field of sdB asteroseismology, the possible impact of higher-order rotation and tidal distortions effects on the pulsation frequencies for this fairly rapidly rotating pulsator with a very close companion. Our main results are summarized as follows:

1. Our additional high-sensitivity time-averaged spectroscopy led us to estimate that the sdB star in PG 1336-018 has $T_{\text {eff }}=32780 \pm 200 \mathrm{~K}, \log g=5.76 \pm 0.03$, and $\log N(\mathrm{He}) / N(\mathrm{H})=-2.94 \pm 0.14$, in excellent agreement with the former, but less accurate, estimation derived by Kilkenny et al. (1998) (see Table 2). These values disagree, except for $\log N(\mathrm{He}) / N(\mathrm{H})$, with the measurement provided by Vučković et al. (2007) whose Echelle spectrum is known to have order-matching problems, however.

2. The seismic search in the vast model parameter space, guided by the above spectroscopic constraints and further considerations on the model properties and the derived mode identification, led us to isolate one optimal model that best matches simultaneously the 25 periods observed in PG 1336-018 and provides a particularly strong internal consistency. This optimal model allowed us to estimate the structural parameters for the sdB star as given in Table 2. In particular, we found an outstanding agreement with the second model proposed by Vučković et al. (2007) characterized by a mass $M=0.466 \pm 0.006 M_{\odot}$ and a radius $R=0.15 \pm 0.01 R_{\odot}$, while our totally independent asteroseismic solution leads to $M=0.459 \pm 0.005 M_{\odot}$ and $R=0.151 \pm 0.001 R_{\odot}$. Such an agreement obtained at this high level of accuracy between two completely independent ways of measuring the mass and radius of that star is quite outstanding and obviously constitutes a crucial test for the validity of both the asteroseismic and the binary light curve modeling methods.

3. The period match for our optimal model solution is excellent, as the observed periods are fitted with an average relative dispersion of $\sim 0.17 \%$ to their associated theoretical periods. On an absolute scale, this represents an average dispersion of $0.27 \mathrm{~s}$ or $10.5 \mu \mathrm{Hz}$ only. Of course, this is still about one order of magnitude larger than the actual precision of the observations, indicating that there is still room for improvements in the modeling of the internal structure of these stars, which is one of our long term goals for $\mathrm{sdB}$ asteroseismology.

4. Our derived mode identification indicates that low-order acoustic modes of degrees $\ell=0,1,2,4$ and radial-orders $k=0-3$ are observed in PG 1336-018. Several groups of periods clearly form multiplets of modes induced by rotational splitting. A follow-up nonadiabatic pulsation calculation applied to this optimal model shows that all the modes associated with the observed periods are indeed expected to be excited through the $\kappa$-mechanism at work in EC 14026 stars (Charpinet et al. 1997). Hence, PG 1336-018 conforms to that requirement as all other EC 14026 pulsators studied in detail thus far and adds another confirmation - if need be - that the mechanism generating the oscillations is correctly identified. We note also that our derived mode identification preserves the apparent amplitude trends expected for modes of increasing degree $\ell$ : on average, the identified $\ell=4$ modes are found to have significantly lower amplitudes than the $\ell=0,1$, and 2 modes, the latter decreasing with increasing $\ell$, as expected. All these elements indicate that a strong internal consistency has been achieved also at the level of the mode identification with the seismic optimal model uncovered for PG 1336-018. We note that independent techniques such as multicolor photometry and time-series spectroscopy could provide interesting independent checks to this proposed mode identification and an additional test on the robustness of the structural parameters and rotation properties inferred from our seismic solution.

5. A remarkable implication of the above mentioned mode identification is that it carries strong indication, from the amplitude of the fitted $\ell=1$ modes distributed among the $m$-components, that the pulsation axis is aligned with the rotation axis of the sdB star. This is the first element of proof ever gathered concerning this geometrical aspect of pulsations and it goes against the idea that the pulsation axis may be tipped toward the companion star due to tidal interactions as proposed by Reed et al. (2006).

6. By relaxing, for the identified model solution (in terms of stellar parameters), the originally imposed solid body rotation law, we further demonstrated that the pulsation spectrum of PG 1336-018 indeed bears the signature of an internal rotation profile that corresponds to a spin-orbit-synchronized star rotating solidly from the surface down to approximately half its stellar radius, at least. We found that the seismic inversion of the rotation profile looses rapidly its accuracy below $r \sim 0.55 R_{\odot}$, as the $p$-mode sensitivity to the deeper regions greatly diminishes. Complete solid rotation cannot be formally confirmed or ruled out, but we provide a strong asteroseismic evidence that the star is indeed most likely tidally locked to its close companion down to $\sim 0.55 R_{\odot}$, which encloses completely the H-rich envelope and the upper part of the He radiative core of the star. This result may be particularly useful to help clarifying the persistent problems related to spin-orbit synchronism through tidal dissipation processes in hot radiative stars (Claret et al. 1995; Claret \& Cunha 1997, and references therein). We also note that if $p$-modes mainly probe the outermost layers of the star, $g$-modes on the contrary are sensitive to the structure and dynamics of much deeper regions and may allow much deeper inversions. In this context, the potential of the long period $\mathrm{sdB}$ pulsators is very high and observations from space, using for instance the French satellite CoRoT and possibly the NASA mission KEPLER, may provide wonderful opportunities in this area.

7. Finally, we demonstrated that higher-order perturbation effects due to rotation and tidal distortion on a pulsating star like PG 1336-018 are, strictly speaking, significant mostly up to second-order with a typical impact of $\sim 10-15 \mu \mathrm{Hz}$ on the mode frequencies. Compared to the achieved accuracy of our best fit model $(\sim 10.5 \mu \mathrm{Hz}$ on average with a standard deviation around that mean of $\sim 9.4 \mu \mathrm{Hz}$ ) limited to first-order rotation corrections, higher-order effects are unlikely, at the outset, to have any significant impact on the determination of the structural and dynamical properties derived for this star at the present level of accuracy. We point out, however, that future studies aimed at improving the physical modeling of PG 1336-018 in order to tighten further the seismic constraints will absolutely need to incorporate accurately the second-order corrections, at the very least. 
We conclude by pointing out that the seismic analysis of PG 1336-018 has led us much farther than anticipated in the early stages of this study. The list of results summarized just above constitutes, in our view, a strong demonstration of the validity and relevance of the forward modeling approach that we have been developing, these past few years, in the specific context of pulsating subdwarf B stars. This approach can, in principle, be generalized to other pulsating stars as well. In this context, our results go against misgivings about the forward approach that developed historically at an epoch when computing ressources were still limited. Of utmost interest here, our forward modeling approach has passed a fundamental consistency test through the comparison with the results of the independent analysis of the binary light curve of PG 1336-018. We suggest, through this example, that asteroseismology can truly live up to its potential. It is the tool of choice for studying in greater detail the internal dynamics and structure of various types of stars.

Acknowledgements. This work made extensive use of the computing facilities offered by the Calcul en Midi-Pyrénées (CALMIP) project and by the Centre Informatique National de l'Enseignement Supérieur (CINES), France. Numerical experiments presented in this paper were also carried out using the Grid'5000 experimental testbed, an initiative from the French Ministry of Research through the ACI GRID incentive action, INRIA, CNRS and RENATER, and other contributing partners (see http://www.grid5000.fr). This work was also supported in part by the NSERC of Canada. G.F. gratefully acknowledges the contribution of the Canada Research Chair Program.

\section{References}

Billères, M., Fontaine, G., Brassard, P., et al. 2000, ApJ, 530, 441 Brassard, P., \& Charpinet, S. 2008, Ap\&SS, 6

Brassard, P., Pelletier, C., Fontaine, G., \& Wesemael, F. 1992, ApJS, 80, 725

Brassard, P., Fontaine, G., \& Wesemael, F. 1995, ApJS, 95, 545

Brassard, P., Fontaine, G., Billères, M., et al. 2001, ApJ, 563, 1013

Briquet, M., Morel, T., Thoul, A., et al. 2007, MNRAS, 381, 1482

Brown, T. M., Ferguson, H. C., Davidsen, A. F., \& Dorman, B. 1997, ApJ, 482, 685

Busso, G., Moehler, S., Zoccali, M., Heber, U., \& Yi, S. K. 2005, ApJ, 633, L29 Charpinet, S., Fontaine, G., Brassard, P., \& Dorman, B. 1996, ApJ, 471, L103

Charpinet, S., Fontaine, G., Brassard, P., et al. 1997, ApJ, 483, L123

Charpinet, S., Fontaine, G., Brassard, P., \& Dorman, B. 2000, ApJS, 131, 223

Charpinet, S., Fontaine, G., \& Brassard, P. 2001, PASP, 113, 775

Charpinet, S., Fontaine, G., \& Brassard, P. 2003, in NATO ASIB Proc. 105: White Dwarfs, ed. D. de Martino, R. Silvotti, J.-E. Solheim, \& R. Kalytis, 69 Charpinet, S., Fontaine, G., Brassard, P., et al. 2005a, A\&A, 443, 251

Charpinet, S., Fontaine, G., Brassard, P., Green, E. M., \& Chayer, P. 2005b, A\&A, 437, 575

Charpinet, S., Silvotti, R., Bonanno, A., et al. 2006, A\&A, 459, 565

Charpinet, S., Fontaine, G., Brassard, P., et al. 2007, Commun. Asteroseism., 150,241

Claret, A., \& Cunha, N. C. S. 1997, A\&A, 318, 187

Claret, A., Gimenez, A., \& Cunha, N. C. S. 1995, A\&A, 299, 724

D’Cruz, N. L., Dorman, B., Rood, R. T., \& O'Connell, R. W. 1996, ApJ, 466, 359

Dorman, B., Rood, R. T., \& O’Connell, R. W. 1993, ApJ, 419, 596

Drechsel, H., Heber, U., Napiwotzki, R., et al. 2001, A\&A, 379, 893

Dupret, M.-A., Thoul, A., Scuflaire, R., et al. 2004, A\&A, 415, 251

Fontaine, G., Brassard, P., Charpinet, S., et al. 2003, ApJ, 597, 518
Fontaine, G., Brassard, P., Charpinet, S., \& Chayer, P. 2006a, Mem. Soc. Astron. It., 77,49

Fontaine, G., Brassard, P., Charpinet, S., et al. 2006b, in ESA SP, 624, Proceedings of SOHO 18/GONG 2006/HELAS I, Beyond the spherical Sun Geier, S., Nesslinger, S., Heber, U., et al. 2007, A\&A, 464, 299

Geier, S., Nesslinger, S., Heber, U., et al. 2008, A\&A, 477, L13

Green, R. F., Schmidt, M., \& Liebert, J. 1986, ApJS, 61, 305

Green, E. M., Liebert, J. W., \& Saffer, R. A. 1997, in The Third Conference on Faint Blue Stars, 417

Green, E. M., Fontaine, G., Reed, M. D., et al. 2003, ApJ, 583, L31

Han, Z., Podsiadlowski, P., Maxted, P. F. L., Marsh, T. R., \& Ivanova, N. 2002, MNRAS, 336, 449

Han, Z., Podsiadlowski, P., Maxted, P. F. L., \& Marsh, T. R. 2003, MNRAS, 341, 669

Heber, U. 1986, A\&A, 155, 33

Heber, U., Reid, I. N., \& Werner, K. 2000, A\&A, 363, 198

Hubeny, I., \& Lanz, T. 1995, ApJ, 439, 875

Kilkenny, D., Koen, C., O’Donoghue, D., \& Stobie, R. S. 1997, MNRAS, 285, 640

Kilkenny, D., O’Donoghue, D., Koen, C., Lynas-Gray, A. E., \& van Wyk, F. 1998, MNRAS, 296, 329

Kilkenny, D., Keuris, S., Marang, F., et al. 2000, The Observatory, 120, 48

Kilkenny, D., Reed, M. D., O'Donoghue, D., et al. 2003, MNRAS, 345, 834

Lanz, T., \& Hubeny, I. 1995, ApJ, 439, 905

Ledoux, P. 1951, ApJ, 114, 373

Lignières, F., Rieutord, M., \& Reese, D. 2006, A\&A, 455, 607

Maxted, P. F. L., Heber, U., Marsh, T. R., \& North, R. C. 2001, MNRAS, 326, 1391

Menzies, J. W. \& Marang, F. 1986, in Instrumentation and Research Programmes for Small Telescopes, ed. J. B. Hearnshaw, \& P. L. Cottrell, IAU Symp., 118, 305

Morales-Rueda, L., Maxted, P. F. L., Marsh, T. R., North, R. C., \& Heber, U. 2003, MNRAS, 338, 752

Orosz, J. A. \& Wade, R. A. 1999, MNRAS, 310, 773

Østensen, R., Oreiro, R., Drechsel, H., et al. 2007, in 15th European Workshop on White Dwarfs, ed. R. Napiwotzki, \& M. R. Burleigh, ASP Conf. Ser., 372, 483

Pamyatnykh, A. A., Handler, G., \& Dziembowski, W. A. 2004, MNRAS, 350, 1022

Randall, S. K., Fontaine, G., Brassard, P., \& Bergeron, P. 2005a, ApJS, 161, 456

Randall, S. K., Matthews, J. M., Fontaine, G., et al. 2005b, ApJ, 633, 460

Randall, S. K., Fontaine, G., Charpinet, S., et al. 2006a, ApJ, 648, 637

Randall, S. K., Fontaine, G., Green, E. M., et al. 2006b, ApJ, 643, 1198

Randall, S. K., Green, E. M., Fontaine, G., et al. 2006c, ApJ, 645, 1464

Randall, S. K., Green, E. M., Van Grootel, V., et al. 2007, A\&A, 476, 1317

Reed, M. D., \& the Whole Earth Telescope XCov21 and 23 collaborations 2006, Baltic Astron., 15, 269

Reese, D., Lignières, F., \& Rieutord, M. 2006, A\&A, 455, 621

Saffer, R. A., Bergeron, P., Koester, D., \& Liebert, J. 1994, ApJ, 432, 351

Saio, H. 1981, ApJ, 244, 299

Tassoul, J.-L., \& Tassoul, M. 1992a, ApJ, 395, 259

Tassoul, M., \& Tassoul, J.-L. 1992b, ApJ, 395, 604

Tremblay, P.-E., Fontaine, G., Brassard, P., Bergeron, P., \& Randall, S. K. 2006, ApJS, 165, 551

Unno, W., Osaki, Y., Ando, H., Saio, H., \& Shibahashi, H. 1989, Non Radial Oscillation of Stars, 2nd edn. (University of Tokyo Press)

Van Grootel, V., Charpinet, S., Fontaine, G., \& Brassard, P. 2008a, A\&A, 483, 875

Van Grootel, V., Charpinet, S., Fontaine, G., et al. 2008b, A\&A, 488, 685

Vučković, M., Aerts, C., Östensen, R., et al. 2007, A\&A, 471, 605

Wesemael, F., Fontaine, G., Bergeron, P., Lamontagne, R., \& Green, R. F. 1992, AJ, 104, 203

Wood, J. H., Zhang, E.-H., \& Robinson, E. L. 1993, MNRAS, 261, 103

Zahn, J.-P. 1977, A\&A, 57, 383 